COMMUNICATIONS IN

ANALYSIS AND GEOMETRY

Volume 13, Number 4, 633-670, 2005

\title{
Foliations by constant mean curvature tubes
}

\author{
Rafe Mazzeo ${ }^{1}$ and Frank Pacard
}

\section{Introduction.}

Constant mean curvature hypersurfaces constitute a very important class of submanifolds in a compact Riemannian manifold $\left(M^{n+1}, g\right)$. In this paper, we are interested in families of such submanifolds, with mean curvature varying from one member of the family to another, which form (partial) foliations and which 'condense' to a submanifold $\Gamma \subset M$ of codimension greater than 1. Our main results concern the existence of such families and, conversely, the geometric nature of the submanifolds $\Gamma$ to which such families can condense.

The simplest case, where $\Gamma$ is a point, was considered by Ye a decade ago, [12], [13]. He proved that if $p \in M$ is a non-degenerate critical point of the scalar curvature function $R_{g}$, then there exists a neighborhood $\mathcal{U} \ni p$ such that $\mathcal{U} \backslash\{p\}$ is foliated by constant mean curvature (for short CMC) spheres; in fact, the members of this family are small perturbations of the geodesic spheres of radius $\rho, 0<\rho<\rho_{0}$, and they have mean curvatures $H=1 / \rho \rightarrow \infty$. Moreover, this foliation is essentially unique. Conversely, if a neighbourhood of $p$ admits such a foliation, then necessarily, $\left.\nabla R_{g}\right|_{p}=0$. In very closely related work, Ye [14], and by quite different methods (using inverse mean curvature flow) Huisken and Yau [4], proved the existence of a unique foliation by CMC spheres near infinity in an asymptotically flat manifold (of non-negative scalar curvature); this is of interest in general relativity.

In this paper, we study the existence of families of CMC hypersurfaces which converge to a (closed, embedded) submanifold $\Gamma^{\ell} \subset M^{n+1}$, particularly in the case $\ell=1$. Define the geodesic tube

$$
\mathcal{T}_{\rho}(\Gamma):=\left\{q \in M^{n+1}: \quad \operatorname{dist}_{g}(q, \Gamma)=\rho\right\} ;
$$

this is a smooth hypersurface provided $\rho$ is smaller than the radius of curvature of $\Gamma$, and we henceforth always tacitly assume that this is the case.

\footnotetext{
${ }^{1}$ Supported in part by NSF under grant \#DMS-0204730
} 
The mean curvature of this tube satisfies

$$
H_{\mathcal{T}_{\rho}(\Gamma)}=\frac{n-\ell}{n \rho}+\mathcal{O}(1) \quad \text { as } \quad \rho \searrow 0
$$

and hence, it is plausible that we might be able to perturb this tube to a CMC hypersurface with $H \equiv(n-\ell) /(n \rho)$. It turns out that this may not be possible for every (small) $\rho>0$, but we prove the:

Theorem 1.1. Suppose that $\Gamma$ is a simple closed embedded geodesic with non-degenerate Jacobi operator. Then, there exist $k_{0} \in \mathbb{N}$ and sequences $\rho_{k}^{\prime}<\rho_{k}^{\prime \prime} \rightarrow 0$, for $k \geq k_{0}$ such that when $\rho \in I_{k}:=\left(\rho_{k}^{\prime}, \rho_{k}^{\prime \prime}\right)$, the geodesic tube $\mathcal{T}_{\rho}(\Gamma)$ may be perturbed to a CMC hypersurface $\Sigma_{\rho}$ with $H=\frac{n-1}{n \rho}$. The $\Sigma_{\rho}$ are non-intersecting and foliate the open set equal to their union, hence, they form a partial foliation of some neighborhood of $\Gamma$.

The hypersurface $\Sigma_{\rho}$ is a small perturbation of $\mathcal{T}_{\rho}(\Gamma)$ in the sense that it is the normal graph (for some function whose $L^{\infty}$ norm is bounded by a constant times $\rho^{3}$ ) over a small translate of $\Gamma$ (by some translation whose $L^{\infty}$ norm is bounded by a constant times $\rho^{2}$ ), cf. Section 4 for the precise formulation. In addition, we have rather precise information on the location and width of the intervals $I_{k}$ :

$$
\begin{aligned}
\rho_{k}^{\prime}-\frac{\sqrt{n-1} \Lambda}{2 \pi(k+1)} & =\mathcal{O}\left(k^{(9-\alpha) / 4}\right), \\
\rho_{k}^{\prime \prime}-\frac{\sqrt{n-1} \Lambda}{2 \pi k} & =\mathcal{O}\left(k^{(9-\alpha) / 4}\right),
\end{aligned}
$$

for all $\alpha \in(0,1)$ where $\Lambda$ equals the length of $\Gamma$. Note that the nondegeneracy condition on $\Gamma$ is a mild and generic one, and that there are no stringent conditions on the curvature along $\Gamma$, as is the case in Ye's theorem when $\Gamma$ is a point.

The existence of gaps in this foliation stems from the fact that at certain radii, the Jacobi operators on the geodesic tubes $\mathcal{T}_{\rho}(\Gamma)$ become degenerate, and this substantially complicates certain analytic steps in the construction. However, this gap behaviour is genuine, and is linked to a bifurcation phenomenon, as we explain more carefully in Section 5 .

Recall that the index of a compact CMC or minimal submanifold is the number of negative eigenvalues of (the negative of) its Jacobi operator. We estimate the index of the leaves of the partial foliation we construct :

Proposition 1.2. If $\rho \in I_{k}$, then for $k$ sufficiently large,

$$
\operatorname{Index}\left(\Sigma_{\rho}\right)=\operatorname{Index}(\Gamma)+2 k+1 \text {. }
$$


In particular, $\operatorname{Index}\left(\Sigma_{\rho}\right) \rightarrow \infty$ as $\rho \rightarrow 0$. It is easy to show that for a generic metric on $M$, the moduli space of CMC hypersurfaces condensing to $\Gamma$ is a smooth one-dimensional set, i.e. a (possibly infinite) union of curves, and that the index is constant on each component. Thus, Proposition 1.2 shows that the leaves $\Sigma_{\rho}$ for $\rho \in I_{k}$ lie in different components of this moduli space.

The final part of this paper concerns necessary conditions on $\Gamma$ in order that a sequence of CMC surfaces condensing to $\Gamma$ exists. We show that if there exists a sequence of CMC hypersurfaces $\Sigma_{j}$, each of which can be written (in an appropriate sense) as a normal graph over the geodesic tube $\mathcal{T}_{\rho_{j}}(\Gamma)$ where $\rho_{j} \rightarrow 0$, then $\Gamma$ must be minimal. We defer to Section 5.2 for the precise statement of this result. We have proved this converse only under rather stringent conditions, but posit the following:

Conjecture: Let $\Gamma$ be a closed embedded $\ell$-dimensional submanifold of $M$ and that there exist sequences $\rho_{k}^{\prime}<\rho_{k}^{\prime \prime} \rightarrow 0$ and a partial foliation by $C M C$ hypersurfaces $\Sigma_{\rho}, \rho \in I_{k}:=\left(\rho_{k}^{\prime}, \rho_{k}^{\prime \prime}\right)$ (with $\left.\rho_{k}^{\prime \prime}<\rho_{k-1}^{\prime}\right)$, satisfying:

(i) The mean curvature of $\Sigma_{\rho}$ equals $\frac{n-1}{n \rho}$;

(ii) There exists a constant $c>0$, independent of $k$ and $\rho \in I_{k}$ such that

$$
\Sigma_{\rho} \subset\left\{q \in M^{n+1}: \quad \operatorname{dist}_{g}(q, \Gamma) \leq c \rho\right\}
$$

(iii) The norm of the second fundamental form of these hypersurfaces satisfy $\left|A_{\Sigma_{\rho}}\right| \leq c \frac{1}{\rho}$ for some constant $c>0$, again independent of $k$ and $\rho \in I_{k}$.

Then, $\Gamma$ is a minimal submanifold.

One might even be able to weaken hypotheses (ii) and (iii) substantially, but even with these hypotheses, the proof is already probably difficult. We have chosen to prove this converse only under much stronger hypotheses in order to include one main calculation which explains why the minimality of $\Gamma$ is the natural conclusion.

On the other hand, the method of proof in this paper encounters serious analytic difficulties when $\operatorname{dim} \Gamma>1$; these complications are ultimately due to the fact that $\left(-\Delta_{\Gamma}-\lambda\right)^{-1}$ is not uniformly bounded when $\lambda \rightarrow \infty$ and $\lambda$ lies in a spectral gap, precisely because these spectral gaps typically decrease in size in this higher dimensional setting. Since this paper was written, we have been able to overcome this and extend our result to the case when $\Gamma$ is a non-degenerate minimal submanifold of arbitrary dimension, see [9]; one of the main new ideas in this extension is an iteration method for improving the initial approximate solution. 
There are some parallels between our Theorem 1 and some recent results concerning solutions of the equation

$$
\varepsilon^{2} \Delta u+f(u)=0
$$

with vanishing Neumann data on a smooth, bounded domain $\Omega \subset \mathbb{R}^{n}$. For example, the second author and Ritoré [10] prove the existence of positive solutions to

$$
\varepsilon^{2} \Delta u-u^{3}+u=0
$$

which concentrate along a minimal submanifold $\Sigma$ as $\varepsilon \searrow 0$. On the other hand, Malchiodi and Montenegro [7] construct positive solutions of

$$
\varepsilon^{2} \Delta u+u^{3}-u=0
$$

which concentrate along $\partial \Omega$ as $\varepsilon \searrow 0$. As in the present paper, the same 'spectral gap' phenomenon arises to domains $\Omega \subset \mathbb{R}^{2}$.

\section{Geometry of tubes.}

In this section, we derive expansions as $\rho \searrow 0$ for the metric, second fundamental form and mean curvature of the tubes $\mathcal{T}_{\rho}(\Gamma)$ and their perturbations. There is a famous and beautiful formula for the volume of these tubes, due originally to Herman Weyl, when $M$ has constant curvature, which has found applications in fields as diverse as geometric measure theory and statistics. We refer to Gray's monograph [3] for Weyl's formula and references to later work.

\subsection{Fermi coordinates and Taylor expansion of the metric near $\Gamma$.}

We first consider the asymptotic development of the metric $g$ in Fermi coordinates around $\Gamma$. This leads to an asymptotic formula for the metric on the geodesic tubes $\mathcal{T}_{\rho}(\Gamma)$. These computations are standard, and are described more systematically in [3].

Fix an arclength parametrization $\gamma(t)$ of $\Gamma, t \in[a, b]:=I$, and denote by $S N \Gamma$ the sphere bundle in $N \Gamma$. Then,

$$
S N \Gamma \ni(t, v) \longmapsto \exp _{\gamma(t)}(\rho v) \in \mathcal{T}_{\rho}(\Gamma)
$$

is a diffeomorphism when $\rho$ is small enough. Choose a parallel orthonormal frame $E_{1}, \cdots, E_{n}$ for $N \Gamma$ (along $(a, b)$, say). This determines a coordinate system

$$
x:=\left(x_{0}, x_{1} \ldots, x_{n}\right) \longmapsto \exp _{\gamma\left(x_{0}\right)}\left(x_{1} E_{1}+\ldots+x_{n} E_{n}\right):=F(x),
$$


and the corresponding coordinate vector fields $X_{\alpha}:=F_{*}\left(\partial_{x_{\alpha}}\right)$. We write $x^{\prime}=$ $\left(x_{1}, \ldots, x_{n}\right)$, and adopt the convention that indices $i, j, k, \ldots \in\{1, \ldots, n\}$, whereas $\alpha, \beta, \ldots \in\{0, \ldots, n\}$.

Remark 2.1. For simplicity, we identify the metric $g$ on $M$ and its pullback $F^{*} g$ on some neighbourhood in $\mathbb{R} \times \mathbb{R}^{n}$. This allows us to use the linear operations on the latter space. With slight abuse of notations, we identify $F\left(x_{0}, x^{\prime}\right)$ with $\left(x_{0}, x^{\prime}\right)$ and $X_{\alpha}$ with $\partial_{x_{\alpha}}$.

We also use cylindrical Fermi coordinates. Thus, let $r=\sqrt{x_{1}^{2}+\ldots+x_{n}^{2}}$, which by Gauss' lemma is the geodesic distance from $x$ to $\Gamma$. The vector

$$
\partial_{r}=\frac{1}{r} \sum_{i=1}^{n} x_{i} X_{i}
$$

is the unit normal to the geodesic tubes.

We have arranged that the metric coefficients $g_{\alpha \beta}=\left\langle X_{\alpha}, X_{\beta}\right\rangle$ equal $\delta_{\alpha \beta}$ along $\Gamma$. We now compute higher terms in the Taylor expansions of these functions. In the following, the notation $\mathcal{O}\left(r^{m}\right)$ indicates a function $f$ such that it and its partial derivatives of any order, with respect to the vector fields $X_{0}$ and $x_{i} X_{j}$, are bounded by $C r^{m}$ in some fixed tubular neighborhood of $\Gamma$. Also, we shall compute the metric coefficients at a point $q:=F\left(x_{0}, x^{\prime}\right)$ in terms of geometric data at $p:=F\left(x_{0}, 0\right)$ and the radius $r=d(p, q)$.

We begin with the expansion of the covariant derivative:

Lemma 2.2. For $\alpha, \beta=0, \ldots, n$,

$$
\nabla_{X_{\alpha}} X_{\beta}=\sum_{\gamma=0}^{n} \mathcal{O}(r) X_{\gamma}
$$

and for $\alpha=\beta=0$, we record the more precise expansion

$$
\nabla_{X_{0}} X_{0}=-\sum_{i, j=1}^{n}\left\langle R\left(X_{j}, X_{0}\right) X_{i}, X_{0}\right\rangle_{p} x_{i} X_{j}+\sum_{\gamma=0}^{n} \mathcal{O}\left(r^{2}\right) X_{\gamma} .
$$

Proof. Anywhere on $\Gamma$,

$$
\nabla_{X_{0}} X_{0}=\nabla_{X_{0}} X_{j}=\nabla_{X_{j}} X_{0}=\nabla_{X_{i}} X_{j}=0 .
$$


The vanishing of the first two terms is obvious since $\Gamma$ is a geodesic and the $X_{i}$ are parallel along it. Because we are using coordinate vector fields, $\nabla_{X_{\alpha}} X_{\beta}=\nabla_{X_{\beta}} X_{\alpha}$ for any $\alpha, \beta$, even away from $\Gamma$, and this implies the vanishing of the third term. Since any $X \in N_{p} \Gamma$ is tangent to the geodesic $\exp _{p}(s X)$, and so $\nabla_{X_{i}+X_{j}}\left(X_{i}+X_{j}\right)=0$ at $p$, hence $\nabla_{X_{i}} X_{j}+\nabla_{X_{j}} X_{i}=0$ there. Combined with the symmetry statement, we obtain that the final term also vanishes. This now gives (2.2).

Next, using (2.2), we get along $\Gamma$

$$
\begin{aligned}
X_{i}\left\langle\nabla_{X_{0}} X_{0}, X_{j}\right\rangle & =\left\langle\nabla_{X_{i}} \nabla_{X_{0}} X_{0}, X_{j}\right\rangle+\left\langle\nabla_{X_{0}} X_{0}, \nabla_{X_{i}} X_{j}\right\rangle \\
& =\left\langle\nabla_{X_{i}} \nabla_{X_{0}} X_{0}, X_{j}\right\rangle \\
& =\left\langle R\left(X_{i}, X_{0}\right) X_{0}, X_{j}\right\rangle+\left\langle\nabla_{X_{0}} \nabla_{X_{i}} X_{0}, X_{j}\right\rangle \\
& =\left\langle R\left(X_{i}, X_{0}\right) X_{0}, X_{j}\right\rangle
\end{aligned}
$$

This implies (2.3).

Our next result gives the expansion of the metric coefficients in Fermi coordinates. The expansion of the $g_{i j}, i, j=1, \ldots, n$, agrees with the well known expansion for the metric in normal coordinates, cf. [11], [6] or [16], but we briefly recall the proof here for completeness.

Proposition 2.3. In the same notation as above, we have

$$
\begin{aligned}
& g_{i j}(q)=\delta_{i j}+\frac{1}{3}\left\langle R\left(X_{k}, X_{i}\right) X_{\ell}, X_{j}\right\rangle_{p} x_{k} x_{\ell}+\mathcal{O}\left(r^{3}\right) \\
& g_{0 i}(q)=\mathcal{O}\left(r^{2}\right) \\
& g_{00}(q)=1+\left\langle R\left(X_{k}, X_{0}\right) X_{\ell}, X_{0}\right\rangle_{p} x_{k} x_{\ell}+\mathcal{O}\left(r^{3}\right) .
\end{aligned}
$$

Proof. The function

$$
X_{k} g_{\alpha \beta}=\left\langle\nabla_{X_{k}} X_{\alpha}, X_{\beta}\right\rangle+\left\langle X_{\alpha}, \nabla_{X_{k}} X_{\beta}\right\rangle
$$

vanishes on $\Gamma$, and thus, the first order terms vanish in all of these Taylor expansions.

To compute the second order terms, it suffices to compute

$$
\begin{aligned}
X_{k} X_{k} g_{\alpha \beta}(p) & =X_{k} X_{k}\left\langle X_{\alpha}, X_{\beta}\right\rangle \\
& =\left\langle\nabla_{X_{k}}^{2} X_{\alpha}, X_{\beta}\right\rangle+\left\langle X_{\alpha}, \nabla_{X_{k}}^{2} X_{\beta}\right\rangle+2\left\langle\nabla_{X_{k}} X_{\alpha}, \nabla_{X_{k}} X_{\beta}\right\rangle
\end{aligned}
$$


and then polarize (i.e. replace $X_{k}$ by $X_{k}+X_{\ell}$, etc.). By (2.2), the final term vanishes along $\Gamma$. Also,

$$
\nabla_{X_{k}}^{2} X_{\alpha}=\nabla_{X_{k}} \nabla_{X_{\alpha}} X_{k}=\nabla_{X_{\alpha}} \nabla_{X_{k}} X_{k}+R\left(X_{k}, X_{\alpha}\right) X_{k}
$$

First let $\alpha=j \geq 1$ and compute the first term on the right. Since $\nabla_{X} \nabla_{X} X=0$ on $\Gamma$ for any $X$ which is a constant linear combination of the $X_{i}$, we have

$$
0=\nabla_{X_{k}+\varepsilon X_{j}} \nabla_{X_{k}+\varepsilon X_{j}}\left(X_{k}+\varepsilon X_{j}\right)
$$

equating the coefficient of $\varepsilon$ to 0 gives $\nabla_{X_{j}} \nabla_{X_{k}} X_{k}=-2 \nabla_{X_{k}} \nabla_{X_{k}} X_{j}$, and hence,

$$
3 \nabla_{X_{k}}^{2} X_{j}=R\left(X_{k}, X_{j}\right) X_{k}
$$

so finally, along $\Gamma$

$$
X_{k} X_{k} g_{i j}=\frac{2}{3}\left\langle R\left(X_{k}, X_{i}\right) X_{k} \cdot X_{j}\right\rangle .
$$

The formula for the second order Taylor coefficient for $g_{i j}$ now follows at once.

When $\alpha=0, \nabla_{X_{0}} \nabla_{X_{k}} X_{k} \equiv 0$ on $\Gamma$, so

$$
\nabla_{X_{k}}^{2} X_{0}=R\left(X_{k}, X_{0}\right) X_{k} .
$$

from which it follows that

$$
X_{k} X_{k} g_{00}=2\left\langle R\left(X_{k}, X_{0}\right) X_{k}, X_{0}\right\rangle
$$

and this gives the formula for $g_{00}$.

The second order Taylor coefficient for $g_{0 i}$ has not been given because it is not needed later.

\subsection{Perturbed tubes and their mean curvature.}

We now describe a suitable class of deformations of the geodesic tubes $\mathcal{T}_{\rho}(\Gamma)$, depending on a section $\Phi$ of $N \Gamma$ and a scalar function $w$ on the spherical normal bundle $S N \Gamma$. One of the main technical parts of this paper, which occupies the rest of Section 2, is the computation of the mean curvature of these hypersurfaces, at least asymptotically in $\rho$ and for sufficiently small $\Phi$ and $w$.

The spherical normal bundle is locally trivialized by the map

$$
(a, b) \times S^{n-1} \ni\left(x_{0}, \theta\right) \longmapsto\left(\gamma\left(x_{0}\right), \sum \theta_{j} E_{j}\right) \in S N \Gamma .
$$


Fix $\rho>0$, and define

$$
G\left(x_{0}, \theta\right):=F\left(x_{0}, \rho\left(1+w\left(x_{0}, \theta\right)\right) \theta+\Phi\left(x_{0}\right)\right) ;
$$

the image of this map will be called $\mathcal{T}_{\rho}(w, \Phi)$. Thus, $\mathcal{T}_{\rho}(w, \Phi)$ is obtained by first taking the normal graph of the function $\rho w$ over the tube of radius $\rho$ in $N \Gamma$ and then translating by $\Phi$. In particular,

$$
\mathcal{T}_{\rho}(0,0)=\mathcal{T}_{\rho}(\Gamma)
$$

It will sometimes be useful to calculate using a coordinate system

$$
\mathbb{R}^{n-1} \ni y \mapsto \Upsilon(y) \in S^{n-1},
$$

with associated coordinates vector fields $Y_{j}=\partial_{y_{j}} \Upsilon$. In particular, we regard $G$ as a function of $\left(x_{0}, y\right)$ and write

$$
G\left(x_{0}, y\right):=F\left(x_{0}, \rho\left(1+w\left(x_{0}, y\right)\right) \Upsilon(y)+\Phi\left(x_{0}\right)\right) .
$$

Two different types of Hölder spaces will be used to measure regularity of functions on $S N \Gamma$ and sections of $N \Gamma$ : first, we use the ordinary Hölder spaces $\mathcal{C}^{m, \alpha}(S N \Gamma), \mathcal{C}^{m, \alpha}(\Gamma, N \Gamma)$, but we shall also use modified Hölder spaces $\mathcal{C}_{\rho}^{m, \alpha}(S N \Gamma), \mathcal{C}_{\rho}^{m, \alpha}(\Gamma, N \Gamma)$ which are based on differentiations with respect to the vector fields $\rho \partial_{x_{0}}$ and $\partial_{y_{j}}$ (where $y$ is any local coordinate system on $S^{n-1}$, see above). Note that this is tantamount to using the rescaled variable $s=x_{0} / \rho$ since $\partial_{s}=\rho \partial_{x_{0}}$. We shall assume that

$$
\Phi\left(x_{0}\right)=\sum_{j=1}^{n} \phi_{j}\left(x_{0}\right) X_{j} \in \mathcal{C}^{2, \alpha}(\Gamma, N \Gamma), \quad w \in \mathcal{C}_{\rho}^{2, \alpha}(S N \Gamma)
$$

For $p \in \Gamma$, let $S_{p}$ denote the spherical fibre of $S N \Gamma$ over $p$. Any function $w$ on $S N \Gamma$ decomposes into a sum of three terms

$$
w=w_{0}+\hat{w}+\tilde{w}
$$

where the restriction to any $S_{p}$ of each of these terms lies in the span of the eigenfunctions $\varphi_{j}(\theta)$ on $S^{n-1}$ with $j=0, j=1, \ldots, n$, and $j>n$, respectively. The first component, $w_{0}$, is a function on $\Gamma$ itself. Next, the eigenfunctions $\varphi_{j}, 1 \leq j \leq n$, are the restrictions to $S^{n-1}$ of linear functions on $\mathbb{R}^{n}$, so any linear combination of them can be identified with a translation in $\mathbb{R}^{n}$ (the linear function $x \rightarrow a \cdot x$ being identified with the translation $x \rightarrow x+a)$. Correspondingly, the summand $\hat{w}$ is canonically associated to a section $\Phi$ of the normal bundle $N \Gamma$. 
We shall typically assume that the functions $w$ has 'linear component' $\hat{w} \equiv 0$, and shall regard the linear part of the perturbation as a section of $N \Gamma$, as just described.

It will be fundamental in the analysis below to regard $w$ as a function of $s=x_{0} / \rho$ and $y_{j}$, but $\Phi$ and $\gamma$ as functions of $x_{0}$ (in particular, whenever we write $\Phi^{\prime}$, we mean $\left.\partial_{x_{0}} \Phi:=\sum \partial_{x_{0}} \phi_{j}\left(x_{0}\right) X_{j}\right)$. However, we sometimes also write $G=G(s, y)$. For example, the tangent space to $\mathcal{T}_{\rho}(w, \Phi)$ is spanned by the vector fields

$$
\begin{aligned}
& Z_{0}=G_{*}\left(\partial_{s}\right)=\rho\left(X_{0}+\partial_{s} w \Upsilon+\Phi^{\prime}\right), \\
& Z_{j}=G_{*}\left(\partial_{y_{j}}\right)=\rho\left((1+w) Y_{j}+\partial_{y_{j}} w \Upsilon\right), \quad j=1, \ldots, n .
\end{aligned}
$$

Definition 2.4. In the following, $L(w, \Phi)$ denotes any expression which is a linear differential operator (of order at most 2) in $w$ and $\Phi$ which satisfies

$$
\|L(w, \Phi)\|_{\mathcal{C}_{\rho}^{0, \alpha}} \leq c\left(\|w\|_{\mathcal{C}_{\rho}^{2, \alpha}(S N \Gamma)}+\|\Phi\|_{\mathcal{C}^{2, \alpha}(\Gamma, N \Gamma)}\right)
$$

where $c$ is independent of $\rho$. Similarly, $Q(w, \Phi)$ denotes any non-linear differential operator (of order less than or equal to 2) in $w$ and $\Phi$ which vanishes quadratically in the pair $(w, \Phi)$ and such that

$$
\begin{aligned}
& \left\|Q\left(w_{2}, \Phi_{2}\right)-Q\left(w_{1}, \Phi_{1}\right)\right\|_{\mathcal{C}_{\rho}^{0, \alpha}} \leq c \sup _{i=1,2}\left(\left\|w_{i}\right\|_{\mathcal{C}_{\rho}^{2, \alpha}(S N \Gamma)}+\left\|\Phi_{i}\right\|_{\mathcal{C}^{2, \alpha}(\Gamma, N \Gamma)}\right) \\
& (2.7) \times\left(\left\|w_{2}-w_{1}\right\|_{\mathcal{C}_{\rho}^{2, \alpha}(S N \Gamma)}+\left\|\Phi_{2}-\Phi_{1}\right\|_{\mathcal{C}^{2, \alpha}(\Gamma, N \Gamma)}\right)
\end{aligned}
$$

Here, the spaces $\mathcal{C}_{\rho}^{0, \alpha}$ are either equal to $\mathcal{C}_{\rho}^{0, \alpha}(S N \Gamma)$ or $\mathcal{C}_{\rho}^{0, \alpha}(\Gamma, N \Gamma)$ according to the range of $L$ and $Q$. Finally, terms denoted $\mathcal{O}\left(\rho^{k}\right)$ are bounded in $\mathcal{C}^{m, \alpha}(S N \Gamma)$ or $\mathcal{C}^{m, \alpha}(\Gamma, N \Gamma)$ by $C \rho^{k}$, where the constant $C$ does not depend on $(w, \Phi)$ or $\rho$.

\subsection{The first fundamental form.}

The next step is the computation of the coefficients of the first fundamental form of $\mathcal{T}_{\rho}(w, \Phi)$ with respect to the coordinates $(s, y)$. At the point

$$
q=F(\rho s, \rho(1+w(s, y)) \Upsilon(y)+\Phi(\rho s))
$$


(where $p=F(\rho s, 0)$ ), we obtain directly from (2.4) that

$$
\begin{aligned}
\left\langle X_{0}, X_{0}\right\rangle_{q}= & 1+\mathcal{O}\left(\rho^{2}\right)+\rho L(w, \Phi)+Q(w, \Phi) \\
\left\langle X_{i}, X_{j}\right\rangle_{q}= & \left\langle X_{i}, X_{j}\right\rangle_{p}+\frac{\rho^{2}}{3}\left\langle R\left(\Upsilon, X_{i}\right) \Upsilon, X_{j}\right\rangle_{p}+\mathcal{O}\left(\rho^{3}\right) \\
& +\frac{\rho}{3}\left[\left\langle R\left(\Upsilon, X_{i}\right) \Phi, X_{j}\right\rangle_{p}+\left\langle R\left(\Phi, X_{i}\right) \Upsilon, X_{j}\right\rangle_{p}\right] \\
& +\rho^{2} L(w, \Phi)+Q(w, \Phi) \\
\left\langle X_{i}, X_{0}\right\rangle_{q}= & \mathcal{O}\left(\rho^{2}\right)+\rho L(w, \Phi)+Q(w, \Phi) .
\end{aligned}
$$

We use these expansions to obtain the expansion of the first fundamental form of $\mathcal{T}_{\rho}(\Phi, w)$.

Proposition 2.5. We have

$$
\begin{aligned}
\rho^{-2}\left\langle Z_{0}, Z_{0}\right\rangle_{q}= & 1+\mathcal{O}\left(\rho^{2}\right)+\rho L(w, \Phi)+Q(w, \Phi) \\
\rho^{-2}\left\langle Z_{0}, Z_{j}\right\rangle_{q}= & \mathcal{O}\left(\rho^{2}\right)+L(w, \Phi)+Q(w, \Phi) \\
\rho^{-2}\left\langle Z_{i}, Z_{j}\right\rangle_{q}= & \left\langle Y_{i}, Y_{j}\right\rangle_{p}+\frac{\rho^{2}}{3}\left\langle R\left(\Upsilon, Y_{i}\right) \Upsilon, Y_{j}\right\rangle_{p}+\mathcal{O}\left(\rho^{3}\right) \\
& +2 w\left\langle Y_{i}, Y_{j}\right\rangle_{p}+\frac{\rho}{3}\left[\left\langle R\left(\Upsilon, Y_{i}\right) \Phi, Y_{j}\right\rangle_{p}+\left\langle R\left(\Upsilon, Y_{j}\right) \Phi, Y_{i}\right\rangle_{p}\right] \\
& \rho^{2} L(w, \Phi)+Q(w, \Phi) .
\end{aligned}
$$

Proof. The first two equations are clear. We give more details about how to derive the third estimate since the same argument will be used frequently. First, it follows from (2.8) that

$$
\begin{aligned}
\left\langle\Upsilon, Y_{j}\right\rangle_{q}= & \left\langle\Upsilon, Y_{j}\right\rangle_{p}+\frac{\rho^{2}}{3}\left\langle R(\Upsilon, \Upsilon) \Upsilon, Y_{j}\right\rangle_{p}+\mathcal{O}\left(\rho^{3}\right) \\
& +\frac{\rho}{3}\left[\left\langle R(\Upsilon, \Upsilon) \Phi, Y_{j}\right\rangle_{p}+\left\langle R(\Phi, \Upsilon) \Upsilon, Y_{j}\right\rangle_{p}\right] \\
& +\rho^{2} L(w, \Phi)+Q(w, \Phi) .
\end{aligned}
$$

However, when $w=\Phi=0,\left\langle\Upsilon, Y_{j}\right\rangle_{q}=0$ since $\Upsilon$ is normal and $Y_{j}$ is tangent to $\mathcal{T}_{\rho}(0,0)$ then, so that the sum of the first three terms on the right, which is independent of $w$ and $\Phi$, must also vanish. This, together with the fact that $R(\Upsilon, \Upsilon)=0$ implies that

$$
\left.\left\langle\Upsilon, Y_{j}\right\rangle_{q}=\frac{\rho}{3}\left\langle R(\Phi, \Upsilon) \Upsilon, Y_{j}\right\rangle_{p}+\rho^{2} L(w, \Phi)+Q(w, \Phi)\right)
$$


In particular, we get $\left\langle\Upsilon, Y_{j}\right\rangle_{q}=\rho L(w, \Phi)+Q(w, \Phi)$. The third equation follows directly from this.

\subsection{The normal vector field.}

The next task is to find expansions for the unit normal to $\mathcal{T}_{\rho}(w, \Phi)$. We begin with the preparatory

Lemma 2.6. The following expansions hold

$$
\begin{aligned}
\langle\Upsilon, \Upsilon\rangle_{q} & =1+\rho^{2} L(w, \Phi)+Q(w, \Phi) \\
\left\langle\Upsilon, Z_{0}\right\rangle_{q} & =\rho L(w, \Phi)+\rho Q(w, \Phi) \\
\left\langle\Upsilon, Z_{j}\right\rangle_{q} & =\rho \partial_{y_{j}} w+\frac{\rho^{2}}{3}\left\langle R(\Phi, \Upsilon) \Upsilon, Y_{j}\right\rangle_{p}+\rho^{3} L(w, \Phi)+\rho Q(w, \Phi)
\end{aligned}
$$

Proof. These follow from (2.8). As at the end of the last subsection, we are using that $\langle\Upsilon, \Upsilon\rangle_{q}=1$ when $w=\Phi=0$ and $R(\Upsilon, \Upsilon)=0$ to obtain the first two expansions. The second expansion follows from the fact that $\left\langle\Upsilon, Z_{0}\right\rangle_{q}=$ 0 when $w=\Phi=0$. Finally, to obtain the last expansion, we use that $\left\langle\Upsilon, Z_{j}\right\rangle_{q}=0$ when $w=\Phi=0$ as well as the first expansion and (2.10).

We can now proceed with the expansion of the unit normal vector field to $\mathcal{T}_{\rho}(w, \Phi)$.

Proposition 2.7. The normal vector field $N$ to $\mathcal{T}_{\rho}(w, \Phi)$ has the expansion

$$
\begin{aligned}
N:= & -\Upsilon+\sum_{j=1}^{n-1} \alpha_{j} Y_{j}+(L(w, \Phi)+Q(w, \Phi)) X_{0} \\
& +\sum_{j=1}^{n-1}\left(\rho^{2} L(w, \Phi)+Q(w, \Phi)\right) X_{j}
\end{aligned}
$$

where the coefficients $\alpha_{j}$ are solutions of the system

$$
\sum_{j=1}^{n-1} \alpha_{j}\left\langle Y_{j}, Y_{i}\right\rangle_{p}=\partial_{y_{i}} w+\frac{\rho}{3}\left\langle R(\Phi, \Upsilon) \Upsilon, Y_{i}\right\rangle_{p}
$$

Proof. Define the vector field

$$
\tilde{N}:=-\Upsilon+a_{0} Z_{0}+\sum_{j=1}^{n-1} a_{j} Z_{j}
$$


by choosing the coefficients $a_{\alpha}$ so that that $\tilde{N}$ is orthogonal to all of the $Z_{\alpha}$.

It follows at once from Lemma 2.6 and (2.9) that $\rho a_{\alpha}=L(w, \Phi)+$ $Q(w, \Phi)$ for every $\alpha$. Plugging this back into each of the equations $\left\langle N, Z_{i}\right\rangle_{q}=$ 0 (thus neglecting the orthogonality condition when $\alpha=0$ now), we find that the $a_{j}$ are solutions of the system

$$
\sum_{j=1}^{n-1} a_{j}\left\langle Y_{j}, Y_{i}\right\rangle_{p}=\frac{1}{\rho} \partial_{y_{i}} w+\frac{1}{3}\left\langle R(\Phi, \Upsilon) \Upsilon, Y_{i}\right\rangle_{p}+\rho L(w, \Phi)+\frac{1}{\rho} Q(w, \Phi)
$$

Recall also that $Z_{j}=\rho Y_{j}+\rho L(w, \Phi)$ so that $a_{i} Z_{i}=\alpha_{i} Y_{i}+\rho^{2} L(w, \Phi)+$ $Q(w, \Phi)$. Finally, we have

$$
|\tilde{N}|_{q}=1+\rho^{2} L(w, \Phi)+Q(w, \Phi) .
$$

This gives (2.11), since $N=\tilde{N} /|\tilde{N}|$.

\subsection{The second fundamental form.}

The most arduous step is the computation of the second fundamental form. To simplify the computations below, we henceforth assume that, at the point $\Upsilon(y) \in S^{n-1}$,

$$
\left\langle Y_{i}, Y_{j}\right\rangle_{p}=\delta_{i j} \quad \text { and } \quad \bar{\nabla}_{Y_{i}} Y_{j}=0, \quad i, j=1, \ldots, n-1
$$

(where $\bar{\nabla}$ is the connection on $T S^{n-1}$ ).

Proposition 2.8. The following expansions hold

$$
\begin{aligned}
\rho^{-2}\left\langle N, \nabla_{Z_{0}} Z_{0}\right\rangle_{q}= & \rho\left\langle R\left(\Upsilon, X_{0}\right) \Upsilon, X_{0}\right\rangle_{p}+\mathcal{O}\left(\rho^{2}\right) \\
& -\frac{1}{\rho} \partial_{s}^{2} w-\left\langle\Phi^{\prime \prime}, \Upsilon\right\rangle_{p}+\left\langle R\left(\Upsilon, X_{0}\right) \Phi, X_{0}\right\rangle_{p} \\
& +\rho L(w, \Phi)+\frac{1}{\rho} Q(w, \Phi), \\
\rho^{-2}\left\langle N, \nabla_{Z_{0}} Z_{j}\right\rangle_{q}= & \mathcal{O}(\rho)+\frac{1}{\rho} L(w, \Phi)+\frac{1}{\rho} Q(w, \Phi), \\
\rho^{-2}\left\langle N, \nabla_{Z_{j}} Z_{j}\right\rangle_{q}= & \frac{1}{\rho}+\frac{2}{3} \rho\left\langle R\left(\Upsilon, Y_{j}\right) \Upsilon, Y_{j}\right\rangle_{p}+\mathcal{O}\left(\rho^{2}\right) \\
& -\frac{1}{\rho} \partial_{y_{j}}^{2} w+\frac{1}{\rho} w+\frac{2}{3}\left\langle R\left(\Phi, Y_{j}\right) \Upsilon, Y_{j}\right\rangle_{p} \\
& +\rho L(w, \Phi)+\frac{1}{\rho} Q(w, \Phi)
\end{aligned}
$$




$$
\rho^{-2}\left\langle N, \nabla_{Z_{i}} Z_{j}\right\rangle_{q}=\mathcal{O}(\rho)+\frac{1}{\rho} L(w, \Phi)+\frac{1}{\rho} Q(w, \Phi), \quad i \neq j
$$

Proof. First note that by Lemma 2.2

$$
\left.\nabla_{X_{\alpha}} X_{\beta}\right|_{q}=\sum_{\gamma=0}^{n}(\mathcal{O}(\rho)+L(w, \Phi)+Q(w, \Phi)) X_{\gamma}
$$

since the coordinates of $q$ depend on $w$ and $\Phi$. Hence, as

$$
\nabla_{Z_{\alpha}} X_{\beta}=\sum_{\gamma=0}^{n}\left(\mathcal{O}\left(\rho^{2}\right)+\rho L(w, \Phi)+\rho Q(w, \Phi)\right) X_{\gamma},
$$

which follows from (2.17) and the fact that $Z_{\alpha}=\rho \sum_{\gamma}(1+L(w, \Phi)) X_{\gamma}$.

We will also use that

$$
N+\Upsilon=\sum_{\alpha=0}^{n}(L(w, \Phi)+Q(w, \Phi)) X_{\alpha}
$$

which follows from (2.11). Finally, we will need the expansions

$$
\begin{aligned}
\left\langle\Upsilon, X_{0}\right\rangle_{q} & =\rho L(w, \Phi)+Q(w, \Phi) \\
\left\langle\Upsilon, Y_{j}\right\rangle_{q} & =\rho L(w, \Phi)+Q(w, \Phi)
\end{aligned}
$$

whose proof can be obtained, as in Lemma 2.6, starting from (2.8).

Estimate (2.13): We must expand

$\rho^{-2}\left\langle N, \nabla_{Z_{0}} Z_{0}\right\rangle_{q}=\rho^{-1}\left(\left\langle N, \nabla_{Z_{0}} X_{0}\right\rangle_{q}+\left\langle N, \nabla_{Z_{0}}\left(\partial_{s} w \Upsilon\right)\right\rangle_{q}+\left\langle N, \nabla_{Z_{0}} \Phi^{\prime}\right\rangle_{q}\right)$.

The estimate is broken into three steps:

Step 1. From (2.11) and Lemma 2.6, we get

$$
\begin{aligned}
\langle N, \Upsilon\rangle_{q}= & -\langle\Upsilon, \Upsilon\rangle_{q}+\sum_{j=1}^{n} \alpha_{j}\left\langle Y_{j}, \Upsilon\right\rangle_{q}+(L(w, \Phi)+Q(w, \Phi))\left\langle X_{0}, \Upsilon\right\rangle_{q} \\
& +\sum_{j=1}^{n}\left(\rho^{2} L(w, \Phi)+Q(w, \Phi)\right)\left\langle X_{j}, \Upsilon\right\rangle_{q} \\
= & -1+\rho^{2} L(w, \Phi)+Q(w, \Phi)
\end{aligned}
$$

Substituting $N=-\Upsilon+N+\Upsilon$ gives

$$
\left\langle N, \nabla_{Z_{0}} \Upsilon\right\rangle_{q}=-\frac{1}{2} \partial_{s}\langle\Upsilon, \Upsilon\rangle_{q}+\left\langle N+\Upsilon, \nabla_{Z_{0}} \Upsilon\right\rangle_{q}
$$


by Lemma 2.6

$$
\partial_{s}\langle\Upsilon, \Upsilon\rangle_{q}=\rho^{2} L(w, \Phi)+Q(w, \Phi),
$$

and (2.19) and (2.18) imply

$$
\left\langle N+\Upsilon, \nabla_{Z_{0}} \Upsilon\right\rangle_{q}=\rho^{2} L(w, \Phi)+\rho Q(w, \Phi) .
$$

Collecting these estimates, we get

$$
\left\langle N, \nabla_{Z_{0}} \Upsilon\right\rangle_{q}=\rho^{2} L(w, \Phi)+Q(w, \Phi) .
$$

Hence, we conclude that

$$
\begin{aligned}
\left\langle N, \nabla_{Z_{0}}\left(\partial_{s} w \Upsilon\right)\right\rangle_{q} & =\partial_{s}^{2} w\langle N, \Upsilon\rangle_{q}+\partial_{s} w\left\langle N, \nabla_{Z_{0}} \Upsilon\right\rangle_{q} \\
& =-\partial_{s}^{2} w+Q(w, \Phi)
\end{aligned}
$$

Step 2. Next,

$$
\left\langle N, \nabla_{Z_{0}} \Phi^{\prime}\right\rangle_{q}=\rho\left\langle N, \Phi^{\prime \prime}\right\rangle_{q}+\sum_{j=1}^{n} \partial_{x_{0}} \phi_{j}\left\langle N, \nabla_{Z_{0}} X_{j}\right\rangle_{q}
$$

where $\Phi^{\prime \prime}\left(x_{0}\right):=\sum \partial_{x_{0}}^{2} \phi_{j}\left(x_{0}\right) X_{j}$. From (2.18), we have

$$
\left(\partial_{x_{0}} \phi_{j}\right)\left\langle N, \nabla_{Z_{0}} X_{j}\right\rangle_{q}=\rho^{2} L(w, \Phi)+\rho Q(w, \Phi) .
$$

Also, using the same decomposition of $N$, and employing (2.19) and (2.8),

$$
\begin{aligned}
\left\langle N, \Phi^{\prime \prime}\right\rangle_{q} & =-\left\langle\Upsilon, \Phi^{\prime \prime}\right\rangle_{q}+\left\langle N+\Upsilon, \Phi^{\prime \prime}\right\rangle_{q} \\
& =-\left\langle\Upsilon, \Phi^{\prime \prime}\right\rangle_{q}+Q(w, \Phi) \\
& =-\left\langle\Upsilon, \Phi^{\prime \prime}\right\rangle_{p}+\rho^{2} L(w, \Phi)+Q(w, \Phi)
\end{aligned}
$$

Collecting these gives

$$
\left\langle N, \nabla_{Z_{0}} \Phi^{\prime}\right\rangle_{q}=-\rho\left\langle\Upsilon, \Phi^{\prime \prime}\right\rangle_{p}+\rho^{2} L(w, \Phi)+\rho Q(w, \Phi) .
$$

Step 3. Expanding $Z_{0}$ gives

$$
\left\langle N, \nabla_{Z_{0}} X_{0}\right\rangle_{q}=\rho\left\langle N, \nabla_{X_{0}} X_{0}\right\rangle_{q}+\rho \partial_{s} w\left\langle N, \nabla_{\Upsilon} X_{0}\right\rangle_{q}+\rho \sum_{j=1}^{n} \partial_{x_{0}} \phi_{j}\left\langle N, \nabla_{X_{j}} X_{0}\right\rangle_{q}
$$


With the help of (2.17) and (2.19), we evaluate

$$
\begin{aligned}
\left\langle N, \nabla_{\Upsilon} X_{0}\right\rangle_{q} & =\mathcal{O}(\rho)+L(w, \Phi)+Q(w, \Phi) \\
\left\langle N, \nabla_{X_{j}} X_{0}\right\rangle_{q} & =\mathcal{O}(\rho)+L(w, \Phi)+Q(w, \Phi) \\
\left\langle N+\Upsilon, \nabla_{X_{0}} X_{0}\right\rangle_{q} & =\rho L(w, \Phi)+Q(w, \Phi),
\end{aligned}
$$

and plugging these into (2.21) already gives

$$
\left\langle N, \nabla_{Z_{0}} X_{0}\right\rangle_{q}=-\rho\left\langle\Upsilon, \nabla_{X_{0}} X_{0}\right\rangle_{q}+\rho^{2} L(w, \Phi)+\rho Q(w, \Phi)
$$

Using (2.3) in Lemma 2.2, we get the expansion

$$
\begin{aligned}
\left.\nabla_{X_{0}} X_{0}\right|_{q}= & -\sum_{j=1}^{n} \rho\left\langle R\left(X_{j}, X_{0}\right) \Upsilon, X_{0}\right\rangle_{p} X_{j}+\mathcal{O}\left(\rho^{2}\right) \\
& -\sum_{j=1}^{n}\left\langle R\left(X_{j}, X_{0}\right) \Phi, X_{0}\right\rangle_{p} X_{j}+\rho L(w, \Phi)+Q(w, \Phi),
\end{aligned}
$$

and so,

$$
\begin{aligned}
\left\langle N, \nabla_{Z_{0}} X_{0}\right\rangle_{q}= & \rho^{2} \sum_{j=1}^{n}\left\langle R\left(X_{j}, X_{0}\right) \Upsilon, X_{0}\right\rangle_{p}\left\langle\Upsilon, X_{j}\right\rangle_{q}+\mathcal{O}\left(\rho^{3}\right) \\
& +\rho \sum_{j=1}^{n}\left\langle R\left(X_{j}, X_{0}\right) \Phi, X_{0}\right\rangle_{p}\left\langle\Upsilon, X_{j}\right\rangle_{q} \\
& +\rho^{2} L(w, \Phi)+\rho Q(w, \Phi) .
\end{aligned}
$$

Finally, using (2.8) again, we conclude that

$$
\begin{aligned}
\left\langle N, \nabla_{Z_{0}} X_{0}\right\rangle_{q}= & \rho^{2}\left\langle R\left(\Upsilon, X_{0}\right) \Upsilon, X_{0}\right\rangle_{p}+\mathcal{O}\left(\rho^{3}\right)+\rho\left\langle R\left(\Upsilon, X_{0}\right) \Phi, X_{0}\right\rangle_{p} \\
& +\rho^{2} L(w, \Phi)+\rho Q(w, \Phi)
\end{aligned}
$$

which, together with the results of Step 1 and Step 2, completes the proof of the first estimate.

Estimate (2.14): Decompose

$$
\begin{aligned}
\left\langle N, \nabla_{Z_{0}} Z_{j}\right\rangle_{q}= & \rho\left\langle N, Y_{j}\right\rangle_{q} \partial_{s} w+\rho\langle N, \Upsilon\rangle_{q} \partial_{s} \partial_{y_{j}} w \\
& +\rho(1+w)\left\langle N, \nabla_{Z_{0}} Y_{j}\right\rangle_{q}+\rho\left\langle N, \nabla_{Z_{0}} \Upsilon\right\rangle_{q} \partial_{y_{j}} w .
\end{aligned}
$$

As above, we use (2.19) and (2.20) to estimate

$$
\left\langle N, Y_{j}\right\rangle_{q}=-\left\langle\Upsilon, Y_{j}\right\rangle_{q}+\left\langle N+\Upsilon, Y_{j}\right\rangle_{q}=L(w, \Phi)+Q(w, \Phi)
$$

Similarly, by Lemma 2.6 and (2.19),

$$
\langle N, \Upsilon\rangle_{q}=-1+L(w, \Phi)+Q(w, \Phi)
$$


But now, by (2.19) and (2.18), we have

$$
\left\langle N, \nabla_{Z_{0}} Y_{j}\right\rangle_{q}=\mathcal{O}\left(\rho^{2}\right)+\rho L(w, \Phi)+\rho Q(w, \Phi)
$$

and, as already seen in the estimate of (2.17)

$$
\left\langle N, \nabla_{Z_{0}} \Upsilon\right\rangle_{q}=\rho^{2} L(w, \Phi)+\rho Q(w, \Phi),
$$

and the proof of the estimate follows directly.

Estimates (2.15) and (2.16): Observe that, by Proposition 2.5, we can also write

$$
N=-\Upsilon+\frac{1}{\rho} \sum_{j=1}^{n} \alpha_{j} Z_{j}+\hat{N}
$$

where

$$
\hat{N}=(L(w, \Phi)+Q(w, \Phi)) X_{0}+\sum_{j=1}^{n}\left(\rho^{2} L(w, \Phi)+Q(w, \Phi)\right) X_{j}
$$

Now, write

$$
\begin{aligned}
\left\langle N, \nabla_{Z_{j}} Z_{j^{\prime}}\right\rangle_{q}= & \left\langle N, \nabla_{Z_{j^{\prime}}} Z_{j}\right\rangle_{q} \\
= & -\frac{1}{2}\left(\left\langle\nabla_{Z_{j}} N, Z_{j^{\prime}}\right\rangle_{q}+\left\langle\nabla_{Z_{j^{\prime}}} N, Z_{j}\right\rangle_{q}\right) \\
= & \frac{1}{2}\left(\left\langle\nabla_{Z_{j}} \Upsilon, Z_{j^{\prime}}\right\rangle_{q}+\left\langle\nabla_{Z_{j^{\prime}}} \Upsilon, Z_{j}\right\rangle_{q}\right) \\
& -\frac{1}{2 \rho} \sum_{i=1}^{n}\left(\left\langle\nabla_{Z_{j}}\left(\alpha_{i} Z_{i}\right), Z_{j^{\prime}}\right\rangle_{q}+\left\langle\nabla_{Z_{j^{\prime}}}\left(\alpha_{i} Z_{i}\right), Z_{j}\right\rangle_{q}\right) \\
& +\frac{1}{2}\left(\left\langle\hat{N}, \nabla_{Z_{j}} Z_{j^{\prime}}\right\rangle_{q}+\left\langle\hat{N}, \nabla_{Z_{j^{\prime}}} Z_{j}\right\rangle_{q}\right) \\
& -\frac{1}{2}\left(\partial_{y_{j}}\left\langle\hat{N}, Z_{j^{\prime}}\right\rangle_{q}+\left.\partial_{y_{j^{\prime}}}\left\langle\hat{N}, Z_{j}\right\rangle\right|_{q}\right)
\end{aligned}
$$

Step 1. By (2.17), we can estimate

$$
\begin{aligned}
\nabla_{Z_{j}} Z_{j^{\prime}}= & \rho \partial_{y_{j}} w Y_{j^{\prime}}+\rho \partial_{y_{j}} \partial_{y_{j^{\prime}}} w \Upsilon \\
& +\rho(1+w) \nabla_{Z_{j}} Y_{j^{\prime}}+\rho \partial_{y_{j^{\prime}}} w \nabla_{Z_{j}} \Upsilon \\
= & \left(\mathcal{O}\left(\rho^{3}\right)+\rho^{2} L(w, \Phi)+\rho^{2} Q(w, \Phi)\right) X_{0} \\
& +\sum_{k=1}^{n}\left(\mathcal{O}\left(\rho^{3}\right)+\rho L(w, \Phi)+\rho^{2} Q(w, \Phi)\right) X_{k},
\end{aligned}
$$


Observe that the coefficient of $X_{0}$ is slightly better than the coefficient of the other $X_{k}$ since the first two terms only involve the $X_{k}$. Using this together with (2.22), we conclude that

$$
\left\langle\hat{N}, \nabla_{Z_{j}} Z_{j^{\prime}}\right\rangle_{q}+\left\langle\hat{N}, \nabla_{Z_{j^{\prime}}} Z_{j}\right\rangle_{q}=\rho^{3} L(w, \Phi)+\rho Q(w, \Phi)
$$

Step 2. Next, using (2.22) together with (2.8), we find that

$$
\left.\partial_{y_{j}}\left\langle\hat{N}, Z_{j^{\prime}}\right\rangle\right|_{q}+\left.\partial_{y_{j^{\prime}}}\left\langle\hat{N}, Z_{j}\right\rangle\right|_{q}=\rho^{3} L(w, \Phi)+\rho Q(w, \Phi)
$$

Step 3. We now estimate

$$
A_{j j^{\prime}}:=\left\langle\nabla_{Z_{j}} \Upsilon, Z_{j^{\prime}}\right\rangle_{q}+\left\langle\nabla_{Z_{j^{\prime}}} \Upsilon, Z_{j}\right\rangle_{q}
$$

It is convenient to define

$$
A_{j j^{\prime}}^{\prime}:=\frac{1}{1+w}\left(\left\langle\nabla_{Z_{j}}(1+w) \Upsilon, Z_{j^{\prime}}\right\rangle_{q}+\left\langle\nabla_{Z_{j^{\prime}}}(1+w) \Upsilon, Z_{j}\right\rangle_{q}\right)
$$

It follows from Lemma 2.6 that

$$
A_{j j^{\prime}}=A_{j j^{\prime}}^{\prime}+\rho Q(w, \Phi)
$$

Hence, it is enough to focus on the estimate of $A_{j j^{\prime}}^{\prime}$. To analyze this term, let us revert for the moment and regard $w$ and $\Phi$ as functions of the coordinates $(t, y)$ (rather than $(s, y)$ ), and also consider $\rho$ as a variable instead of just a parameter. Thus, we consider

$$
\tilde{F}(\rho, t, y)=F(t, \rho(1+w(t, y)) \Upsilon(y)+\Phi(t))
$$

The coordinate vector fields $Z_{j}$ are still equal to $\tilde{F}_{*}\left(\partial_{y_{j}}\right)$, but now, we also have $(1+w) \Upsilon=\tilde{F}_{*}\left(\partial_{\rho}\right)$, which is the identity we wish to use below. Now, from $(2.9)$, we write

$$
A_{j j^{\prime}}^{\prime}=\frac{1}{1+w}\left(\left\langle\nabla_{\partial_{\rho}} Z_{j}, Z_{j^{\prime}}\right\rangle_{q}+\left\langle\nabla_{\partial_{\rho}} Z_{j^{\prime}}, Z_{j}\right\rangle_{q}\right)=\left.\frac{1}{1+w} \partial_{\rho}\left\langle Z_{j}, Z_{j^{\prime}}\right\rangle\right|_{q}
$$


Therefore, it follows from (2.9) in Proposition 2.5 that

$$
\begin{aligned}
A_{j j^{\prime}}= & \frac{1}{1+w} \partial_{\rho}\left[\rho^{2}\left\langle Y_{j}, Y_{j^{\prime}}\right\rangle_{p}+\frac{\rho^{4}}{3}\left\langle R\left(\Upsilon, Y_{j}\right) \Upsilon, Y_{j^{\prime}}\right\rangle_{p}+\mathcal{O}\left(\rho^{5}\right)\right. \\
& +2 \rho^{2} w\left\langle Y_{j}, Y_{j^{\prime}}\right\rangle_{p}+\frac{\rho^{3}}{3}\left(\left\langle R\left(\Upsilon, Y_{j}\right) \Phi, Y_{j^{\prime}}\right\rangle_{p}+\left\langle R\left(\Upsilon, Y_{j^{\prime}}\right) \Phi, Y_{j}\right\rangle_{p}\right) \\
& \left.+\rho^{4} L(w, \Phi)+\rho^{2} Q(w, \Phi)\right]+\rho Q(w, \Phi) \\
= & \frac{1}{1+w}\left[2 \rho\left\langle Y_{j}, Y_{j^{\prime}}\right\rangle_{p}+\frac{4}{3} \rho^{3}\left\langle R\left(\Upsilon, Y_{j}\right) \Upsilon, Y_{j^{\prime}}\right\rangle_{p}+\mathcal{O}\left(\rho^{4}\right)\right. \\
& +4 \rho w\left\langle Y_{j}, Y_{j^{\prime}}\right\rangle_{p}+\rho^{2}\left(\left\langle R\left(\Upsilon, Y_{j}\right) \Phi, Y_{j^{\prime}}\right\rangle_{p}+\left\langle R\left(\Upsilon, Y_{j^{\prime}}\right) \Phi, Y_{j}\right\rangle_{p}\right) \\
& \left.+\rho^{3} L(w, \Phi)\right]+\rho Q(w, \Phi) \\
= & 2 \rho\left\langle Y_{j}, Y_{j^{\prime}}\right\rangle_{p}+\frac{4}{3} \rho^{3}\left\langle R\left(\Upsilon, Y_{j}\right) \Upsilon, Y_{j^{\prime}}\right\rangle_{p}+\mathcal{O}\left(\rho^{4}\right) \\
& +2 \rho w\left\langle Y_{j}, Y_{j^{\prime}}\right\rangle_{p}+\rho^{2}\left(\left\langle R\left(\Upsilon, Y_{j}\right) \Phi, Y_{j^{\prime}}\right\rangle_{p}+\left\langle R\left(\Upsilon, Y_{j^{\prime}}\right) \Phi, Y_{j}\right\rangle_{p}\right) \\
& +\rho^{3} L(w, \Phi)+\rho Q(w, \Phi)
\end{aligned}
$$

Step 4. Finally, we must compute

$$
\begin{aligned}
B_{j j^{\prime}}:= & \left\langle\nabla_{Z_{j}}\left(\alpha_{i} Z_{i}\right), Z_{j^{\prime}}\right\rangle_{q}+\left\langle\nabla_{Z_{j^{\prime}}}\left(\alpha_{i} Z_{i}\right), Z_{j}\right\rangle_{q} \\
= & \left\langle Z_{i}, Z_{j^{\prime}}\right\rangle_{q} \partial_{y_{j}} \alpha_{i}+\left\langle Z_{i}, Z_{j}\right\rangle_{q} \partial_{y_{j^{\prime}}} \alpha_{i} \\
& +\alpha_{i}\left(\left\langle\nabla_{Z_{j}} Z_{i}, Z_{j^{\prime}}^{\prime}\right\rangle_{q}+\left\langle\nabla_{Z_{j^{\prime}}} Z_{i}, Z_{j}\right\rangle_{q}\right) \\
= & \left\langle Z_{i}, Z_{j^{\prime}}\right\rangle_{q} \partial_{y_{j}} \alpha_{i}+\left\langle Z_{i}, Z_{j}\right\rangle_{q} \partial_{y_{j^{\prime}}} \alpha_{i} \\
& +\alpha_{i}\left(\left\langle\nabla_{Z_{i}} Z_{j}, Z_{j}^{\prime}\right\rangle_{q}+\left\langle\nabla_{Z_{i}} Z_{j^{\prime}}, Z_{j}\right\rangle_{q}\right) \\
= & \left\langle Z_{i}, Z_{j^{\prime}}\right\rangle_{q} \partial_{y_{j}} \alpha_{i}+\left\langle Z_{i}, Z_{j}\right\rangle_{q} \partial_{y_{j^{\prime}}} \alpha_{i}+\alpha_{i} \partial_{y_{i}}\left\langle Z_{j}, Z_{j^{\prime}}\right\rangle_{q}
\end{aligned}
$$

Observe that (2.12) implies

$$
\partial_{y_{j}}\left\langle Y_{i}, Y_{j^{\prime}}\right\rangle_{p}=0
$$

Using this together with (2.9) and the expression for the $\alpha_{i}$ given in Proposition 2.7 , we get

$$
\alpha_{i} \partial_{y_{i}}\left\langle Z_{j}, Z_{j}^{\prime}\right\rangle_{q}=\rho^{4} L(w, \Phi)+\rho^{2} Q(w, \Phi)
$$

It follows from (2.9) and the definition of $\alpha_{i}$ again that

$$
\left\langle Z_{i}, Z_{j^{\prime}}\right\rangle_{q} \partial_{y_{j}} \alpha_{i}=\rho^{2}\left\langle Y_{i}, Y_{j^{\prime}}\right\rangle_{p} \partial_{y_{j}} \alpha_{i}+\rho^{4} L(w, \Phi)+\rho^{2} Q(w, \Phi)
$$


Therefore, it remains to estimate $\left\langle Y_{i}, Y_{j^{\prime}}\right\rangle_{p} \partial_{y_{j}} \alpha_{i}$. By definition, we have

$$
\sum_{i=1}^{n} \alpha_{i}\left\langle Y_{i}, Y_{j^{\prime}}\right\rangle_{p}=\partial_{y_{j^{\prime}}} w+\frac{\rho}{3}\left\langle R(\Phi, \Upsilon) \Upsilon, Y_{j^{\prime}}\right\rangle_{p}
$$

Differentiating with respect to $y_{j}$, we get

$$
\sum_{i=1}^{n}\left(\left\langle Y_{i}, Y_{j^{\prime}}\right\rangle_{p} \partial_{y_{j}} \alpha_{i}+\alpha_{i} \partial_{y_{j}}\left\langle Y_{i}, Y_{j^{\prime}}\right\rangle_{p}\right)=\partial_{y_{j}} \partial_{y_{j^{\prime}}} w+\frac{\rho}{3} \partial_{y_{j}}\left\langle R(\Phi, \Upsilon) \Upsilon, Y_{j^{\prime}}\right\rangle_{p}
$$

Again, it follows from (2.12) that $\partial_{y_{j}}\left\langle Y_{i}, Y_{j^{\prime}}\right\rangle_{p}=0$.

Moreover, we have

$$
\nabla_{Z_{j}} \Upsilon=Y_{j} \quad \text { and } \quad \nabla_{Z_{j}} Y_{j^{\prime}}=a \Upsilon
$$

for some $a \in \mathbb{R}$. Reinserting this in (2.23) yields

$$
\begin{aligned}
\sum_{i=1}^{n}\left\langle Y_{i}, Y_{j^{\prime}}\right\rangle_{p} \partial_{y_{j}} \alpha_{i}= & \partial_{y_{j}} \partial_{y_{j^{\prime}}} w+\frac{\rho}{3}\left\langle R\left(\Phi, Y_{j}\right) \Upsilon, Y_{j^{\prime}}\right\rangle_{p} \\
& +\frac{\rho}{3}\left\langle R(\Phi, \Upsilon) Y_{j}, Y_{j^{\prime}}\right\rangle_{p},
\end{aligned}
$$

since $R(\Upsilon, \Upsilon)=0$.

Collecting these estimates, we conclude that

$$
B_{j j}=\rho^{2} \partial_{y_{j}}^{2} w+\frac{\rho^{3}}{3}\left\langle R\left(\Phi, Y_{j}\right) \Upsilon, Y_{j}\right\rangle_{p}+\rho^{4} L(w, \Phi)+\rho^{2} Q(w, \Phi)
$$

since $\left\langle R(\Phi, \Upsilon) Y_{j}, Y_{j}\right\rangle_{p}=0$ and also that

$$
B_{j j^{\prime}}=\rho^{2} L(w, \Phi)+\rho^{2} Q(w, \Phi)
$$

when $j \neq j^{\prime}$. With the estimates of the previous steps, this finishes the proof of the last two estimates!

\subsection{The mean curvature.}

Collecting the estimates of the last subsection and taking the trace, we have now proved that the mean curvature $H(w, \Phi)$ of the hypersurface $\mathcal{T}_{\rho}(w, \Phi)$ satisfies

$$
\begin{gathered}
n \rho H(w, \Phi)-(n-1)= \\
\left(\frac{2}{3}\left\langle R\left(\Upsilon, X_{0}\right) \Upsilon, X_{0}\right\rangle_{p}-\frac{1}{3} \operatorname{Ric}(\Upsilon, \Upsilon)\right) \rho^{2}+\mathcal{O}\left(\rho^{3}\right) \\
-\left(\partial_{s}^{2} w+\Delta_{S^{n-1}} w+(n-1) w\right)-\rho\left\langle\Phi^{\prime \prime}+R\left(\Phi, X_{0}\right) X_{0}, \Upsilon\right\rangle_{p} \\
+\rho^{2} L(w, \Phi)+Q(w, \Phi) .
\end{gathered}
$$


(We recall that if $E_{\alpha}$ is an orthonormal basis of $T_{p} M$, then

$$
\left.\operatorname{Ric}(\Upsilon, \Upsilon):=-\sum_{\alpha=0}^{n}\left\langle R\left(\Upsilon, E_{\alpha}\right) \Upsilon, E_{\alpha}\right\rangle_{p} .\right)
$$

Denote by $\left(\lambda_{j}, \varphi_{j}\right)$ the eigendata of $\Delta_{S^{n-1}}$, where the eigenfunctions are orthonormal and counted with multiplicity.

A most important observation is that the second and third terms in the expansion of $n \rho H$ are quadratic in the coordinates $x_{j}$. Hence, when $\Phi=w=0$, we have

$$
\begin{array}{ll}
\left(n \rho H-(n-1), \varphi_{j}\right)_{L^{2}\left(S^{n-1}\right)}=\mathcal{O}\left(\rho^{3}\right), & j=1, \ldots, n, \\
\left(n \rho H-(n-1), \varphi_{j}\right)_{L^{2}\left(S^{n-1}\right)}=\mathcal{O}\left(\rho^{2}\right), & j \neq 1, \ldots, n,
\end{array}
$$

or in other words, writing $f=n \rho H-(n-1)$, then $f=\mathcal{O}\left(\rho^{2}\right)$, but its $L^{2}\left(S^{n-1}\right)$ projection over $\varphi_{1}, \ldots, \varphi_{n}$ satisfies $\hat{f}=\mathcal{O}\left(\rho^{3}\right)$.

\section{Jacobi operators.}

In this section, we examine the mapping properties of some linear operators which appear in the expression of the mean curvature of $\mathcal{T}_{\rho}(w, \Phi)$ given in (2.24).

\subsection{Definitions.}

The two linear operators appearing in the third line of (2.24) are

$$
\begin{array}{ll}
w \longmapsto \mathcal{L}_{S N \Gamma} w & :=\partial_{s}^{2} w+\Delta_{\theta} w+(n-1) w, \\
\Phi \longmapsto \mathcal{J} \Phi & :=\nabla_{X_{0}}^{2} \Phi+R\left(\Phi, X_{0}\right) X_{0} .
\end{array}
$$

The latter is the Jacobi operator on $\Gamma$ corresponding to the second variation of the length functional on curves, while (up to a multiplicative factor) the former appears in the second variation of the area functional about a Euclidean cylinder $\mathbb{R} \times S^{n-1}(\rho)$.

Recall that the geodesic $\Gamma$ is said to be non-degenerate when $\mathfrak{J}$ is invertible, i.e. if the equation $\mathfrak{J} \Phi=0$ has no non-trivial solutions on all of $\Gamma$. For a generic metric on $M$, it is well known that all closed geodesics are non-degenerate.

On the other hand, since it is already naturally expressed in terms of the scaled coordinate $s$,

$$
\mathcal{L}_{S N \Gamma}: \mathcal{C}_{\rho}^{2, \alpha}(S N \Gamma) \longrightarrow \mathcal{C}_{\rho}^{0, \alpha}(S N \Gamma)
$$


is bounded uniformly in $\rho$. We can analyze this operator using the eigendecomposition for $\Delta_{\theta}$ on $S^{n-1}$. As in Section 2.2, if the eigenfunction decomposition of $w$ is given by

$$
w(s, \theta)=\sum_{j \geq 0} w_{j}(s) \varphi_{j}(\theta)
$$

then $w$ decomposes as $w_{0}+\hat{w}+\tilde{w}$, where

$$
\hat{w}:=\sum_{j=1}^{n} w_{j} \varphi_{j} \quad \text { and } \quad \tilde{w}:=\sum_{j>n} w_{j} \varphi_{j} .
$$

We denote by $\Pi_{0}, \hat{\Pi}$ and $\tilde{\Pi}$ the projections on to these three components, respectively. From now on, we assume that we are working with functions $w$ such that $\hat{\Pi} w=0$, and thus, we only need to be concerned with the operators $\left(\mathcal{L}_{S N \Gamma}\right)_{0}$ and $\tilde{\mathcal{L}}_{S N \Gamma}$ induced on the two other components. Note in particular, that

$$
\left(\mathcal{L}_{S N \Gamma}\right)_{0}:=\partial_{s}^{2}+n-1
$$

\subsection{Mapping properties.}

We now study the mapping properties of $\mathfrak{J}$ and (the components of) $\mathcal{L}_{S N \Gamma}$.

We first note that

$$
\mathfrak{J}: \mathcal{C}^{2, \alpha}(\Gamma, N \Gamma) \longrightarrow \mathcal{C}^{0, \alpha}(\Gamma, N \Gamma)
$$

is an isomorphism when $\Gamma$ is a non-degenerate geodesic.

Next, we also assert that

$$
\tilde{\mathcal{L}}_{S N \Gamma}: \tilde{\Pi} \mathcal{C}_{\rho}^{2, \alpha}(S N \Gamma) \longrightarrow \tilde{\Pi} \mathcal{C}_{\rho}^{0, \alpha}(S N \Gamma)
$$

is an isomorphism with inverse uniformly bounded as $\rho \rightarrow 0$; this follows from the fact that $\Delta_{\theta}+(n-1) \leq-C<0$ on this subspace. Details are left to the reader.

Finally, it is clear that

$$
\left(\mathcal{L}_{S N \Gamma}\right)_{0}: \Pi_{0} \mathcal{C}_{\rho}^{2, \alpha}(S N \Gamma) \longrightarrow \Pi_{0} \mathcal{C}_{\rho}^{0, \alpha}(S N \Gamma)
$$

is bounded for every $\rho>0$, but is only invertible when

$$
\sqrt{n-1} \frac{\Lambda}{\rho} \notin 2 \pi \mathbb{N}
$$


in the exceptional cases, there is a two-dimensional nullspace spanned by

$$
\cos (\sqrt{n-1} s), \quad \sin (\sqrt{n-1} s)
$$

and hence, a two-dimensional cokernel. To determine the norm of its inverse when $\sqrt{n-1} \Lambda / \rho \notin 2 \pi \mathbb{Z}$, suppose that $\left(\mathcal{L}_{S N \Gamma}\right)_{0} v=f$. Then,

$$
\begin{aligned}
\sqrt{n-1} v(s)= & \sin (\sqrt{n-1} s)\left(\alpha+\int_{0}^{s} \cos (\sqrt{n-1} \sigma) f(\sigma) d \sigma\right) \\
& -\cos (\sqrt{n-1} s)\left(\beta+\int_{0}^{s} \sin (\sqrt{n-1} \sigma) f(\sigma) d \sigma\right)
\end{aligned}
$$

where the constants $\alpha, \beta$ are chosen so that $v$ is $\Lambda / \rho$-periodic. We find

$$
|\alpha|+|\beta| \leq \frac{c}{\rho(1-\cos (\sqrt{n-1} \Lambda / \rho))}\|f\|_{L^{\infty}(S N \Gamma)}
$$

for some constant $c>0$, independent of $\rho$, and from this, we have

$$
\|v\|_{\mathcal{C}_{\rho}^{2, \alpha}(S N \Gamma)} \leq c\left(\|f\|_{\mathcal{C}_{\rho}^{0, \alpha}(S N \Gamma)}+\frac{1}{\rho(1-\cos (\sqrt{n-1} \Lambda / \rho))}\|f\|_{L^{\infty}(S N \Gamma)}\right),
$$

again for some constant $c>0$ independent of $\rho$. Note that when $f \in \mathcal{C}^{1}$, there is an equivalent formula

$$
\begin{aligned}
(n-1) v(s)-f(s)= & \sin (\sqrt{n-1} s)\left(\alpha-\int_{0}^{s} \sin (\sqrt{n-1} \sigma) \partial_{s} f(\sigma) d \sigma\right) \\
& +\cos (\sqrt{n-1} s)\left(\beta-\int_{0}^{s} \cos (\sqrt{n-1} \sigma) \partial_{s} f(\sigma) d \sigma\right),
\end{aligned}
$$

where again $\alpha, \beta$ are chosen so that $v$ is $\Lambda / \rho$-periodic. We now obtain

$$
|\alpha|+|\beta| \leq \frac{c}{\rho(1-\cos (\sqrt{n-1} \Lambda / \rho))}\left\|\partial_{s} f\right\|_{L^{\infty}(S N \Gamma)}
$$

for some constant $c>0$ independent of $\rho$, so that

$$
\left.\|v\|_{\mathcal{C}_{\rho}^{2, \alpha}(S N \Gamma)} \leq c\left(\|f\|_{\mathcal{C}_{\rho}^{0, \alpha}(S N \Gamma)}+\frac{1}{\rho(1-\cos (\sqrt{n-1} \Lambda / \rho))}\left\|\partial_{s} f\right\|_{L^{\infty}(S N \Gamma)}\right)\right) .
$$




\section{The constant mean curvature foliation.}

We now use the results of Sections 2 and 3 to perturb $\mathcal{T}_{\rho}(\Gamma)$ to a constant mean curvature hypersurface, at least for $\rho$ sufficiently far from values where $\left(\mathcal{L}_{S N \Gamma}\right)_{0}$ is degenerate.

According to the analysis of Section 2, we must find $w \in \mathcal{C}_{\rho}^{2, \alpha}(S N \Gamma)$ and $\Phi \in \mathcal{C}^{2, \alpha}(\Gamma, N \Gamma)$ such that

$$
n \rho H(w, \Phi)=n-1
$$

Let us denote by

$$
f:=\frac{2}{3}\left\langle R\left(\Upsilon, X_{0}\right) \Upsilon, X_{0}\right\rangle_{p} \rho^{2}-\frac{1}{3} \operatorname{Ric}(\Upsilon, \Upsilon) \rho^{2}+\mathcal{O}\left(\rho^{3}\right),
$$

the inhomogeneous term appearing in (2.24) which corresponds to the mean curvature when $w=\Phi=0$. As usual, this decomposes into three components, $f_{0}+\hat{f}+\tilde{f}$, where $\hat{f}$ corresponds to a section of the normal bundle which we write as $\rho \Psi$. We are searching for $w=w_{0}+\tilde{w}$ and $\Phi$ which satisfy the coupled system

$$
\left\{\begin{array}{cc}
\left(\mathcal{L}_{S N \Gamma}\right)_{0} w_{0} & =f_{0}+\rho^{2} L(w, \Phi)+Q(w, \Phi) \\
\mathfrak{J} \Phi & =\Psi+\rho L(w, \Phi)+\frac{1}{\rho} Q(w, \Phi) \\
\tilde{\mathcal{L}}_{S N \Gamma} \tilde{w} & =\tilde{f}+\rho^{2} L(w, \Phi)+Q(w, \Phi)
\end{array}\right.
$$

We use the function space

$$
\mathcal{E}_{\rho}^{2, \alpha}:=\Pi_{0} \mathcal{C}_{\rho}^{2, \alpha}(S N \Gamma) \oplus \mathcal{C}^{2, \alpha}(\Gamma, N \Gamma) \oplus \tilde{\Pi} \mathcal{C}_{\rho}^{2, \alpha}(S N \Gamma),
$$

where, for $\Xi=\left(w_{0}, \Phi, \tilde{w}\right)$,

$\|\Xi\|_{\mathcal{E}_{\rho}^{2, \alpha}}:=(1-\cos (\sqrt{n-1} \Lambda / \rho))\left\|w_{0}\right\|_{\mathcal{C}_{\rho}^{2, \alpha}(S N \Gamma)}+\|\Phi\|_{\mathcal{C}^{2, \alpha}(\Gamma, N \Gamma)}+\|\tilde{w}\|_{\mathcal{C}_{\rho}^{2, \alpha}(S N \Gamma)}{ }$.

The linear operators appearing on the left in (4.2) are all invertible provided $\sqrt{n-1} \Lambda / \rho \notin 2 \pi \mathbb{Z}$. Thus, multiplying by their inverses, we rewrite this system as

$$
\Xi=\mathfrak{N}(\Xi)
$$

and so, we solve our problem by finding a fixed point of $\mathfrak{N}$ in $\mathcal{E}_{\rho}^{2, \alpha}$.

Lemma 4.1. Write $\Xi_{0}:=\mathfrak{N}(0)$. Then, for $\sqrt{n-1} \Lambda / \rho \notin 2 \pi \mathbb{Z}$, we have

$$
\left\|\Xi_{0}\right\|_{\mathcal{E}_{\rho}^{2, \alpha}} \leq \frac{c_{0}}{2} \rho^{2}
$$

for some $c_{0}>0$. 
Proof. Clearly,

$$
\left\|f_{0}\right\|_{\mathcal{C}_{\rho}^{0, \alpha}(S N \Gamma)}+\rho^{-1}\left\|\partial_{s} f_{0}\right\|_{L^{\infty}(S N \Gamma)} \leq c \rho^{2}
$$

moreover

$$
\|\tilde{f}\|_{\mathcal{C}_{\rho}^{0, \alpha}(S N \Gamma)} \leq c \rho^{2} \quad \text { and } \quad\|\Psi\|_{\mathcal{C}^{0, \alpha}(\Gamma, N \Gamma)} \leq c \rho^{2} .
$$

Since by definition,

$$
\Xi_{0}=\left(\left(\mathcal{L}_{S N \Gamma}\right)_{0}^{-1} f_{0}, \mathfrak{J}^{-1} \Psi_{0},\left(\tilde{\mathcal{L}}_{S N \Gamma}\right)^{-1} \tilde{f}\right)
$$

the result follows from (3.3) and the uniform boundedness of the inverses of the inverses of these linear operators as $\rho \rightarrow 0$.

Next, from the properties of the operators $L$ and $Q$, we deduce the

Lemma 4.2. There exists a constant $c>0$ such that, for the same $c_{0}$ as in the previous Lemma, and for any $\Xi_{1}, \Xi_{2} \in \mathcal{E}_{\rho}^{2, \alpha}$ satisfying

$$
\left\|\Xi_{i}\right\|_{\mathcal{E}_{\rho}^{2, \alpha}} \leq c_{0} \rho^{2}
$$

we have

$$
\left\|\mathfrak{N}\left(\Xi_{2}\right)-\mathfrak{N}\left(\Xi_{1}\right)\right\|_{\mathcal{E}_{\rho}^{2, \alpha}} \leq c \frac{\rho^{1-\alpha}}{(1-\cos (\sqrt{n-1} \Lambda / \rho))^{2}}\left\|\Xi_{2}-\Xi_{1}\right\|_{\mathcal{E}_{\rho}^{2, \alpha}}
$$

Proof. It follows from (2.6) that

$$
\left\|\rho^{2} L(w, \Phi)\right\|_{\mathcal{C}_{\rho}^{0, \alpha}} \leq c \frac{\rho^{2}}{1-\cos (\sqrt{n-1} \Lambda / \rho)}\|\Xi\|_{\mathcal{E}_{\rho}^{2, \alpha}}
$$

if $w=w_{0}+\tilde{w}$ and $\Xi=\left(w_{0}, \Phi, \tilde{w}\right)$. Moreover, if $\left\|\Xi_{i}\right\|_{\mathcal{E}_{\rho}^{2, \alpha}} \leq c_{0} \rho^{2}$, then, we have from $(2.7)$

$$
\left\|Q\left(w_{2}, \Phi_{2}\right)-Q\left(w_{1}, \Phi_{1}\right)\right\|_{\mathcal{C}_{\rho}^{0, \alpha}} \leq c \frac{\rho^{2}}{(1-\cos (\sqrt{n-1} \Lambda / \rho))^{2}}\left\|\Xi_{2}-\Xi_{1}\right\|_{\mathcal{E}_{\rho}^{2, \alpha}} .
$$

where $w_{i}=w_{0, i}+\tilde{w}_{i}$ and $\Xi=\left(w_{0, i}, \Phi_{i}, \tilde{w}_{i}\right)$. Now, the result follows at once from the inequality

$$
\|\Phi\|_{\mathcal{C}^{0, \alpha}(\Gamma, N \Gamma)} \leq c \rho^{-\alpha}\|\Phi\|_{\mathcal{C}_{\rho}^{0, \alpha}(\Gamma, N \Gamma)} .
$$

together with the uniform bounds on $\tilde{\mathcal{L}}_{S N \Gamma}^{-1}$ and $\mathfrak{J}^{-1}$, and the bound on $\left(\mathcal{L}_{S N \Gamma}\right)_{0}^{-1}$ given in (3.2). Details are left to the reader.

Collecting these results, we now have the 
Proposition 4.3. Fix $\alpha \in(0,1)$. Then, there exists a $c_{1}>0$ such that if $k$ is sufficiently large and $\rho$ satisfies

$$
\frac{1}{k+1}+\frac{c_{1}}{k^{(9-\alpha) / 4}} \leq \frac{2 \pi}{\sqrt{n-1} \Lambda} \rho \leq \frac{1}{k}-\frac{c_{1}}{k^{(9-\alpha) / 4}},
$$

then there exists a solution $\left(w_{0}, \tilde{w}, \Phi\right)$ of $(4.2)$ in $\mathcal{E}_{\rho}^{2, \alpha}$. This solution satisfies

$$
\left\|\left(w_{0}, \tilde{w}, \Phi\right)\right\|_{\mathcal{E}_{\rho}^{2, \alpha}} \leq c_{0} \rho^{2}
$$

Proof. It is easy to check that

$$
\frac{\rho^{1-\alpha}}{(1-\cos (\sqrt{n-1} \Lambda / \rho))^{2}}
$$

is as small as we want, provided $c_{1}$ is chosen large enough. It is then easy to check that, when $k$ is large enough, $\mathfrak{N}$ is a contraction from the ball of radius $c_{0} \rho^{2}$ into itself.

This proposition yields the existence of CMC perturbations of the tubes $\mathcal{T}_{\rho}(\Gamma)$ for all radii $\rho \in I_{k}$, when $k$ is large. We shall denote the perturbation functions as $w_{\rho}$ and $\Phi_{\rho}$ to emphasize their dependence on $\rho$. We shall now revert to thinking of these as depending on $x_{0}$ rather than $s$, and in particular, we write

$$
w_{\rho}\left(x_{0}, \theta\right):=w_{0}\left(x_{0} / \rho\right)+\tilde{w}\left(x_{0} / \rho, \theta\right) .
$$

Following through the proof, it is not hard to see that these functions depend smoothly on $\rho$. Furthermore, since the tubes $\mathcal{T}_{\rho}(\Gamma)$ already foliate, it suffices to verify that the mapping

$$
\left(\rho, x_{0}, \theta\right) \longmapsto G\left(x_{0}, \rho\left(1+w_{\rho}\right) \theta+\Phi_{\rho}\right)
$$

is a local diffeomorphism.

First,

$$
\left\|w_{\rho}\right\|_{L^{\infty}(S N \Gamma)}+\|\Phi\|_{L^{\infty}(\Gamma, N \Gamma)} \leq c \rho^{2} .
$$

Also, from the construction itself, we have

$$
\left\|\partial_{\rho} w_{\rho}\right\|_{L^{\infty}(S N \Gamma)}+\left\|\partial_{\rho} \Phi\right\|_{L^{\infty}(\Gamma, N \Gamma)} \leq c \rho .
$$

These certainly imply that (4.3) is a local diffeomorphism, and hence, our CMC surfaces form a local foliation. 


\section{Explaining the gaps.}

In special cases, such as when $\Gamma$ is a circle in the flat torus $T^{n+1}=S^{1} \times$ $\mathbb{R}^{n} / a \mathbb{Z}^{n}$, all of the geodesic tubes about $\Gamma$, of any radius, have constant mean curvature, and thus there are no gaps in the local foliation. On the other hand, Theorem 1 only provides for a local foliation with gaps. As indicated in the introduction, there are good reasons why this construction does not work at all radii. We explain this in greater detail now. We first show that for generic metrics, the moduli space of CMC surfaces isotopic to a geodesic tubes $\mathcal{T}_{\rho}(\Gamma)$ in $M \backslash \Gamma$ is smooth and one-dimensional. The index of the Jacobi operator is constant along components, and by estimating the index for the surfaces close to $\mathcal{T}_{\rho}(\Gamma), \rho \in I_{k}$, we show that there are infinitely many components of this moduli space. We conclude by examining in more detail a very degenerate case, where all of the geodesic tubes $\mathcal{T}_{\rho}(\Gamma)$ are CMC (when smooth), so there are no gaps, but we prove that, in this situation, there are infinitely many bifurcating branches.

\subsection{The moduli space.}

Denote by $\mathcal{H}(M, \Gamma, g)$ the moduli space of all $\mathrm{CMC}$ surfaces $\Sigma \hookrightarrow M$ which are isotopic to any one of the geodesic tubes $\mathcal{T}_{\rho}(\Gamma)$, for $\rho$ small, in $M \backslash \Gamma$, with respect to the metric $g$.

Proposition 5.1. There is an open dense set $\mathcal{U}$ of metrics (in the $\mathcal{C}^{m, \alpha}$ topology for any $m \geq 3)$ on $M$ such that for $g \in \mathcal{U}, \mathcal{H}(M, \Gamma, g)$ is a smooth one-dimensional manifold.

Fix a surface $\Sigma_{0}$ in the correct isotopy class, which has CMC with respect to some metric $g_{0}$. Nearby surfaces may be written as normal graphs over $\Sigma_{0}$, and hence are parametrized by (small) scalar functions on $\Sigma_{0}$. Now, consider the mapping

$$
G: \mathcal{C}^{m, \alpha}\left(M, S^{2} T^{*} M\right) \times \mathcal{C}^{m, \alpha}\left(\Sigma_{0}\right) \longrightarrow \mathcal{C}^{m-2, \alpha}\left(\Sigma_{0}\right)
$$

which assigns to a metric $g$ and a scalar function $w$ on $\Sigma_{0}$ the mean curvature function of the submanifold $\Sigma_{w}=\left\{x+w(x) \nu(x): x \in \Sigma_{0}\right\}$, regarded as a function on $\Sigma_{0}$, with respect to $g$. It is not hard to show [15] (and also [8]) that the differential of this mapping is always surjective, and moreover, the restriction of this differential to the tangent space of the second factor (which is simply the Jacobi operator) is Fredholm of index zero, and is an isomorphism except when there exist non-trivial Jacobi fields. The result 
is then a straightforward application of the Sard-Smale theorem, since $G$ is transverse to the one-dimensional curve of constant functions in the range space.

On the other hand, applying the construction of Theorem 1 when the metric $g \in \mathcal{U}$, we obtain a set of smooth one-dimensional families CMC surfaces in $\mathcal{H}(M, \Gamma, g)$. Let $G_{g}(w)=G(g, w)$; an implication of this proposition is that when $G_{g}$ is regular at $w$ and the mean curvature of $\Sigma_{w}$ is equal to $H$, then some interval $(H-\epsilon, H+\epsilon)$ parameterizes $\mathcal{H}(M, \Gamma, g)$ locally near $\Sigma_{w}$, or in other words, the mean curvatures of the CMC surfaces near to $\Sigma_{w}$ assume all values near to $H$. Although in this case the corresponding surfaces form a local foliation, this need not be true in general.

\subsection{The index.}

We now claim that for generic $g, \mathcal{H}(M, \Gamma, g)$ has infinitely many components. In the following, for $\rho \in I_{k}$, let $\Sigma_{\rho}$ denote the CMC hypersurface constructed in Theorem 1.

Proposition 5.2. Let $g$ be a metric for which $\mathcal{H}(M, \Gamma, g)$ is a smooth onedimensional manifold. Then, for each sufficiently large value of $k$, the surfaces $\Sigma_{\rho}, \rho \in I_{k}$, lie in different components of $\mathcal{H}(M, \Gamma, g)$ and have index equal to $\operatorname{Index}(\Gamma)+2 k+1$.

We prove this theorem by computing the index of $\Sigma_{\rho}$; by definition, this is the number of negative eigenvalues of the Jacobi operator $\mathcal{L}_{\rho}$ on $\Sigma_{\rho}$. This index is locally constant in $\mathcal{H}(M, \Gamma, g)$ when this moduli space is nondegenerate, but since we shall show that the index increases with $k$, this will imply the result.

It follows from (2.24) and the properties of solution $\left(w_{0}, \tilde{w}, \Phi\right)$ given in Proposition 4.3 that the Jacobi operator about $\Sigma_{\rho}$, i.e. the linearization of the operator $\left(w_{0}, \tilde{w}, \Phi\right) \mapsto n \rho H$, has the form

$$
\begin{aligned}
\mathcal{L}_{\rho}(v, \Psi)= & -\left(\partial_{s}^{2} v+\Delta_{S^{n-1}} v+(n-1) v\right)-\rho\left\langle\Psi^{\prime \prime}+R\left(X_{0}, \Psi\right) X_{0}, \theta\right\rangle_{p} \\
& +\rho^{2} L(v, \Psi),
\end{aligned}
$$

where the (linear operator) $L$ satisfies the usual assumptions. 
Lemma 5.3. The quadratic form associated to $\mathcal{L}_{\rho}$ has the expansion

$$
\begin{aligned}
B_{\rho}(v, \Psi):= & \int_{S N \Gamma}\left(\left|\partial_{s} v\right|^{2}+\left|\nabla_{\theta} v\right|^{2}-(n-1) v^{2}\right) \\
& +\omega_{n} \int_{\Gamma}\left(\left|\partial_{t} \Psi\right|^{2}-\left\langle R\left(\Psi, X_{0}\right) \Psi, X_{0}\right\rangle_{p}\right) \\
& +\rho^{3 / 2} C(v, \Psi)
\end{aligned}
$$

where $v \in \Pi_{0} H_{\rho}^{1}(S N \Gamma) \oplus \tilde{\Pi} H_{\rho}^{1}(S N \Gamma)$ (i.e. the Sobolev space $H^{1}(S N \Gamma)$ defined with respect to the vector fields $\partial_{s}$ and $\left.\partial_{y_{j}}\right), \Psi \in H^{1}(\Gamma, N \Gamma), \omega_{n}$ is a positive constant depending only on the dimension, and where $C$ is a quadratic form satisfying

$$
|C(v, \Psi)| \leq c\left(\int_{S N \Gamma}\left(\left|\partial_{s} v\right|^{2}+\left|\nabla_{\theta} v\right|^{2}+v^{2}\right)+\frac{1}{\rho} \int_{\Gamma}\left(\left|\partial_{t} \Psi\right|^{2}+|\Psi|^{2}\right)\right)
$$

for some constant $c>0$ independent of $\rho$.

Proof. For $v$ and $\Psi=\sum \psi_{j} E_{j}$ as in this statement, the fibrewise linear function on $S N \Gamma$ corresponding to $\Psi$ is $\hat{v}=\sum \psi_{j} \theta_{j}$. We can either regard $B_{\rho}$ as a quadratic form in the variables $(v, \Psi)$ or in $v+\frac{1}{\rho} \hat{v}$. Then, by definition

$$
B_{\rho}(v+\hat{v})=\int_{S N \Gamma}\left(\mathcal{L}_{\rho}(v, \Psi)\right)\left(v+\frac{1}{\rho} \hat{v}\right)
$$

Inserting the expression (5.1) and integrating over $\Sigma_{\rho}$, we obtain the first summand in the expression for $B_{\rho}$, involving only $v$, without difficulty. Next, integrating over the spherical fibres of $S N \Gamma$, we have

$$
-\int_{S N \Gamma}\left\langle\Psi^{\prime \prime}, \theta\right\rangle\langle\Psi, \theta\rangle=\sum_{j, k=1}^{n} \int_{S N \Gamma} \psi_{j}^{\prime} \psi_{k}^{\prime} \theta_{j} \theta_{k}=\omega_{n} \sum_{j=1}^{n} \int_{\Gamma}\left(\psi_{j}^{\prime}\right)^{2} ;
$$

there is a similar reduction for the term in $\mathfrak{J}$ of order 0 to an integral over $\Gamma$. The error term leading to $C$ arises from the error term in $\mathcal{L}_{\rho}$, as well as the discrepancy in this last calculation caused by using the volume form on $S N \Gamma$ rather than the one on $\Sigma_{\rho}$. The first of these error terms,

$$
\int L(v, \Psi)\left(v+\frac{1}{\rho} \hat{v}\right)
$$

is almost of the correct form. However, since $\mathcal{L}_{\rho}$ involves $\Psi^{\prime \prime}$, this error might include terms of the form $v \Psi^{\prime \prime}$, which are at first glance too big, 
since, integrating by parts, they equal $\rho^{-1} \partial_{s} v \Psi^{\prime}$. However, examining the computations leading up to (2.24), one can check that $\Phi^{\prime \prime}$ only enters through the term

$$
\rho\left\langle N, \Phi^{\prime \prime}\right\rangle_{p}\left(1+\mathcal{O}\left(\rho^{2}\right)+L(w, \Phi)+Q(w, \Phi)\right)
$$

(recall that we compute $\rho$ times the mean curvature), which has linearization

$$
\rho\left\langle N, \Psi^{\prime \prime}\right\rangle_{p}\left(1+\mathcal{O}\left(\rho^{2}\right)+L(w, \Phi)+Q(w, \Phi)\right)=\rho\left\langle\Psi^{\prime \prime}, \theta\right\rangle_{p}+\mathcal{O}\left(\rho^{3}\right) L(\Psi) .
$$

Hence, this gives, at worst, terms like $\rho^{2} \partial_{s} v \Psi^{\prime}$. It is much more straightforward to check that all the other terms in $C$ satisfy the correct bounds.

As usual, write $v(s, y)=v_{0}(s)+\tilde{v}(s, y)$, where both summands are orthogonal to the linear eigenfunctions $\varphi_{j}, 1 \leq j \leq n$; we also identify $\hat{v}(t, y):=\langle\Psi, \theta\rangle_{p}$. Thus, for $v=v_{0}+\frac{1}{\rho} \hat{v}+\tilde{v} \in \Pi_{0} H_{\rho}^{1}(S N \Gamma) \oplus \hat{\Pi} H^{1}(S N \Gamma) \oplus$ $\tilde{\Pi} H_{\rho}^{1}(S N \Gamma)$, we have the quadratic form

$$
B_{\rho}(v)=B_{0}\left(v_{0}\right)+\hat{B}(\hat{v})+\tilde{B}(\tilde{v})+\rho^{2} C(v),
$$

where

$$
\begin{aligned}
B_{0}\left(v_{0}\right) & :=\omega_{n} \int_{\Gamma}\left(\left|\partial_{s} v_{0}\right|^{2}-(n-1) v_{0}^{2}\right) \\
\hat{B}(\hat{v}) & :=\omega_{n} \int_{\Gamma}\left(\left|\partial_{t} \Psi\right|^{2}-\left\langle R\left(\Psi, X_{0}\right) \Psi, X_{0}\right\rangle_{p}\right) \\
\tilde{B}(\tilde{v}) & :=\int_{S N \Gamma}\left(\left|\partial_{s} \tilde{v}\right|^{2}+\left|\nabla_{\theta} \tilde{v}\right|^{2}-(n-1) \tilde{v}^{2}\right)
\end{aligned}
$$

Assuming that $\rho \in I_{k}=\left(\rho_{k}^{\prime}, \rho_{k}^{\prime \prime}\right)$, let us now compute the index of $B$. Since this index is locally constant in $\rho$, we shall choose

$$
\rho=\frac{\sqrt{n-1}}{4 \pi} \Lambda\left(\frac{1}{k}+\frac{1}{k+1}\right)
$$

which is directly in the middle of $I_{k}$.

Clearly,

$$
\int\left|\partial_{s} \tilde{v}\right|^{2}+|\nabla \tilde{v}|^{2}+\tilde{v}^{2} \leq c \tilde{B}(\tilde{v})
$$

Next, decompose $\hat{v}=\hat{v}^{+}+\hat{v}^{-}$, where $\hat{v}^{ \pm}$lies in the sum of the eigenspaces of $\mathfrak{J}$ with positive or negative eigenvalues, respectively. (Recall that this operator is assumed to be non-degenerate, hence has no zero eigenspace.) We then have

$$
\int\left|\partial_{t} \hat{v}^{+}\right|^{2}+\left|\hat{v}^{+}\right|^{2} \leq c \hat{B}\left(\hat{v}^{+}\right)
$$


and

$$
\int\left|\partial_{t} \hat{v}^{-}\right|^{2}+\left|\hat{v}^{-}\right|^{2} \leq-c \hat{B}\left(\hat{v}^{-}\right) .
$$

We can similarly decompose the remaining component $v_{0}$ as $v_{0}^{+}+v_{0}^{-}$, where $v_{0}^{ \pm}$lie in the eigenspaces corresponding to the positive or negative eigenvalues of $\left(\mathcal{L}_{S N \Gamma}\right)_{0}=\partial_{s}^{2}+(n-1)$. Using that $\rho$ lies in the middle of $I_{k}$, we can estimate

$$
\int\left|\partial_{s} v_{0}^{+}\right|^{2}+\left|v_{0}^{+}\right|^{2} \leq c \rho^{-1} B_{0}\left(v_{0}^{+}\right)
$$

and

$$
\int\left|\partial_{s} v_{0}^{-}\right|^{2}+\left|v_{0}^{-}\right|^{2} \leq-c \rho^{-1} B_{0}\left(v_{0}^{-}\right)
$$

Using all of these estimates, we now obtain that

$$
\begin{aligned}
|C(v)| \leq & c \rho^{-1}\left(B_{0}\left(v_{0}^{+}\right)-B_{0}\left(v_{0}^{-}\right)\right) \\
& +c \rho^{-1}\left(\hat{B}\left(\hat{v}^{+}\right)-\hat{B}\left(\hat{v}^{-}\right)\right)+c \tilde{B}(\tilde{v})
\end{aligned}
$$

for some constant $c>0$ independent of $\rho$. This gives, in turn,

$$
B^{\prime}(v) \leq B_{\rho}(v) \leq B^{\prime \prime}(v)
$$

where

$$
\begin{aligned}
B^{\prime \prime}(v)= & (1+c \rho)\left(B_{0}\left(v_{0}^{+}\right)+\rho \hat{B}\left(\hat{v}^{+}\right)\right) \\
& +(1-c \rho)\left(B\left(v_{0}^{-}\right)+\rho \hat{B}\left(\hat{v}^{-}\right)\right) \\
& +\left(1+c \rho^{2}\right) \tilde{B}(\tilde{v})
\end{aligned}
$$

and

$$
\begin{aligned}
B^{\prime}(v)= & \left(1-c \rho^{1 / 2}\right)\left(B_{0}\left(v_{0}^{+}\right)+\hat{B}\left(\hat{v}^{+}\right)\right) \\
& +\left(1+c \rho^{1 / 2}\right)\left(B\left(v_{0}^{-}\right)+\hat{B}\left(\hat{v}^{-}\right)\right) \\
& +\left(1+c \rho^{3 / 2}\right) \tilde{B}(\tilde{v}) .
\end{aligned}
$$

These upper and lower bounds on $B$ imply that

$$
\operatorname{Index}\left(B^{\prime \prime}\right) \leq \operatorname{Index}\left(B_{\rho}\right) \leq \operatorname{Index}\left(B^{\prime}\right) .
$$

The proof is completed by the following:

Lemma 5.4. When $\rho$ is small enough, the index of $B^{\prime}$ and $B^{\prime \prime}$ are both equal to $\operatorname{Index}(\Gamma)+2 k+1$. 
Proof. If $\rho$ is chosen so that $1-c \rho>1 / 2$, then the index of $B^{\prime}$ and $B^{\prime \prime}$ equals the sum of the dimensions of the spaces on which $B_{0}$ and $\hat{B}$ are negative. But these equal $2 k+1$ and Index $(\Gamma)$, respectively.

\subsection{Bifurcations in a degenerate case.}

We consider in more detail the (very) degenerate case where $(M, g)$ is the flat torus $T^{n+1}=S^{1} \times \mathbb{R}^{n} / a \mathbb{Z}^{n}, a>0$ and $\Gamma=S^{1} \times\{0\}$. After moding out by all the continuous symmetries, the moduli space is still one-dimensional, but has infinitely many singularities.

Each of the geodesic tubes $\mathcal{T}_{\rho}(\Gamma):=\Sigma_{\rho}=S^{1} \times S^{n-1}(\rho)$ is CMC, with mean curvature $H_{\rho}=(n-1) / \rho$. We only consider the case where $\Sigma_{\rho}$ is embedded, i.e. when $\rho<a / 2$. The Jacobi operator for $\Sigma_{\rho}$ is

$$
L_{\rho}=\Delta_{\Sigma_{\rho}}+\left|A_{\Sigma_{\rho}}\right|^{2} .
$$

In terms of our standard cylindrical coordinates, $t \in S^{1}, \theta \in S^{n-1}$,

$$
L_{\rho}=\partial_{t}^{2}+\frac{1}{\rho^{2}}\left(\Delta_{\theta}+(n-1)\right)
$$

Introducing eigendata $\left\{\phi_{k}(t), k^{2}\right\}$ and $\left\{\psi_{\ell}(\theta), \lambda_{\ell}^{2}\right\}$ in each component, we see that $L_{\rho}$ reduces to multiplication by $B(k, \ell, \rho)=-k^{2}+\rho^{-2}\left(n-1-\lambda_{\ell}^{2}\right)$ on the $(k, \ell)$ eigenspace. Since $\lambda_{\ell}^{2}$ is always of the form $j(n-2+j)$ for some non-negative integer $j$, we have $n-1-\lambda_{\ell}^{2} \leq 0$ unless $\ell=0$. This gives the

Proposition 5.5. The surface $\Sigma_{\rho}$ is always degenerate; its nullspace consists of the span of the eigenmodes $\phi_{0}(t) \psi_{\ell}(\theta), \ell=1, \ldots, n$ and, in case $\rho^{2}=(n-1) / k^{2}$ for some $k \in \mathbb{N}$, also $\phi_{k}(t) \psi_{0}(\theta)$.

The 'trivial degeneracies' are those comprised by the first set of elements, which exist for all $\rho$. These correspond to the obvious geometric fact that translating $\Sigma_{\rho}$ parallel to itself in any direction normal to $\Gamma$ gives a family of CMC surfaces with the same mean curvature. These can be eliminated if we mod out by these symmetries. Namely, using linear coordinates $x=$ $\left(x_{0}, x_{1}, \ldots, x_{n}\right)$ in $T^{n+1}$, let $G$ be the finite group generated by reflections in the $x_{j}=0$ plane, $j=1, \ldots, n$.

Corollary 5.6. Acting on the space of $G$-invariant functions on $S N \Gamma$, the Jacobi operator $L_{\rho}$ is degenerate if and only if $\rho^{2}=(n-1) / k^{2}, k \in \mathbb{N}$. 
These degeneracies have a direct geometric explanation too, for it is precisely at these radii where the family of ' $k$-bump' Delaunay surfaces begins to develop.

We now have the picture that $\mathcal{H}\left(T^{n+1}, S^{1}, g_{0}\right)$ consists of the union of the interval $(0, a / 2)_{\rho}$ and infinitely many other intervals $\left(0,(n-1) / k^{2}\right]_{\varepsilon_{k}}$, where the variable $\varepsilon_{k}$ is the Delaunay necksize in the $k^{\text {th }}$ bifurcating branch. In other words, the moduli space looks like an open interval with infinitely many spines sticking out of it. When the metric on $T^{n+1}$ is perturbed generically, this moduli space smooths out; the singularity at each degenerate radius disappears, and this 'spiny interval' breaks into infinitely many components. The CMC surfaces for these slightly perturbed metrics are small perturbations either of the geodesic tubes or else of the Delaunay surfaces, except near the turning points. The gaps encountered in our construction correspond exactly to the small regions around these degenerate radii where the moduli space is curving away from the interval $(0, a / 2)_{\rho}$.

\section{Limits of constant mean curvature foliations.}

There is a sort of converse to Theorem 1.1 which we can prove regardless of the dimension of $\Gamma$. Let $\Gamma$ be a closed $\ell$-dimensional submanifold of $M^{n+1}$, $1 \leq \ell \leq n$, and suppose that there exists a sequence of hypersurfaces $\Sigma_{j}$ such that

a) $\Sigma_{j}$ has constant mean curvature $(n-\ell) / n \rho_{j}$, where $\left\{\rho_{j}\right\}$ is a decreasing sequence with $\rho_{j} \searrow 0$;

b) $\Sigma_{j}$ is isotopic in $M \backslash \Gamma$ to the tube $\mathcal{T}_{\rho_{j}}(\Gamma)$;

c) There exists a $c>0$ such that $\Sigma_{j}$ is contained inside $\mathcal{T}_{c \rho_{j}}(\Gamma)$ for all $j$.

Item c) implies that $\Sigma_{j} \rightarrow \Gamma$ in Hausdorff distance; note also that we are not requiring the existence of any sort of local foliation, just a sequence of $\mathrm{CMC}$ hypersurfaces converging to $\Gamma$. We conjecture that some set of hypotheses very near to these (for example, assuming also a bound on the second fundamental form, as in the introduction, or that $\Sigma_{j}$ is trapped between the tubes of radius $c^{\prime} \rho_{j}$ and $c \rho_{j}$ for fixed constants $0<c^{\prime}<c$.) should be enough to ensure that $\Gamma$ is minimal. Unfortunately, this seems to be quite difficult to prove, and so we shall restrict ourselves to a very special situation by making a fourth, quite restrictive, hypothesis:

d) There exist functions $w_{j} \in \mathcal{C}^{2, \alpha}(S N \Gamma), \Phi_{j} \in \mathcal{C}^{2, \alpha}(\Gamma, N \Gamma)$ satisfying

$$
\left\|w_{j}\right\|_{\mathcal{C}^{2, \alpha}}+\left\|\Phi_{j}\right\|_{\mathcal{C}^{2, \alpha}} \leq c \rho_{j}^{2}
$$


for some $c>0$, independent of $\rho_{j}$, such that

$$
\Sigma_{j}=\mathcal{T}_{\rho_{j}}\left(w_{j}, \Phi_{j}\right)
$$

Quite important (and potentially restrictive) here is that the norms of $w_{j}$ and $\Phi_{j}$ are bounded in $\mathcal{C}^{2, \alpha}, \operatorname{not} \mathcal{C}_{\rho}^{2, \alpha}$.

Theorem 6.1. Let $\Gamma$ be a $\mathcal{C}^{2}$ compact embedded $\ell$-dimensional submanifold of $M$ for some $1 \leq \ell \leq n$, and suppose that $\Sigma_{j}$ is a sequence of CMC hypersurfaces converging to $\Gamma$ and satisfying the hypotheses a) $-d$ ). Then, $\Gamma$ is minimal.

The proof is based on an argument from geometric measure theory which is now fairly standard in the analysis of such 'condensation problems', cf. [1], [2].

We drop the subscript $j$ and consider a functional for which each $\Sigma_{\rho}$ is critical. The argument proceeds by writing the formula which expresses the fact that the first variation of this functional vanishes, and then taking the limit of this formula (in a very weak sense) as $\rho \rightarrow 0$. The limiting first variation equation implies the minimality of $\Gamma$.

Any one of the CMC hypersurfaces $\Sigma=\Sigma_{\rho}$ bound a compact domain $D(\Sigma)$, which is the component of $M \backslash \Sigma$ containing $\Gamma$. Define the measure

$$
d \mu_{\Sigma}=d A_{\Sigma}-n H d V_{\Sigma}
$$

where $H$ is the (constant) mean curvature of $\Sigma$ and where we have set

$$
d A_{\Sigma}:=\mathcal{H}^{n}\left\lfloor_{\Sigma} \quad \text { and } \quad d V_{\Sigma}:=\mathcal{H}^{n+1}\left\lfloor_{D(\Sigma)}\right.\right.
$$

( $\mathcal{H}^{k}$ is $k$ dimensional Hausdorff measure).

CMC hypersurfaces are critical for the functional $\Sigma \rightarrow \int d \mu_{\Sigma}$. In other words, if $X$ is any $\mathcal{C}^{2}$ vector field on $M$, and $\phi_{t}$ the associated one-parameter family of diffeomorphisms, then

$$
\left.\int d \mu_{\phi_{t}^{*} \Sigma}\right|_{t=0}=0
$$

We now compute this variation another way. In fact, for any hypersurface $\Sigma$ and any continuous function $f$, we derive that

$$
\left.\partial_{t} \int f d A_{\phi_{t}^{*} \Sigma}\right|_{t=0}=\int X f d A_{\Sigma}+\int f\left(\operatorname{div} X-\left\langle\nabla_{N} X, N\right\rangle\right) d A_{\Sigma},
$$


where $N$ is the unit normal to $\Sigma$, and similarly,

$$
\left.\partial_{t} \int f d V_{\phi_{t}^{*} \Sigma}\right|_{t=0}=\int X f d V_{\Sigma}+\int f \operatorname{div} X d V_{\Sigma}
$$

Hence, setting $f \equiv 1$ and using (6.1), we obtain

$$
0=\left.\partial_{t} \int d \mu_{\phi_{t}^{*} \Sigma}\right|_{t=0}=\int \operatorname{div} X d \mu_{\Sigma}-\int\left\langle\nabla_{N} X, N\right\rangle d A_{\Sigma} .
$$

Next, let $d L_{\Gamma}=\mathcal{H}^{\ell}\left\lfloor_{\Gamma}\right.$ and denote by $\omega_{n-\ell}$ the volume of $S^{n-\ell}$ with respect to its standard metric (thus $\omega_{n-\ell} /(n+1-\ell)$ is the volume of the ball $\left.B^{n+1-\ell}\right)$.

Lemma 6.2. As $\rho \searrow 0$,

$$
\rho^{\ell-n} d A_{\Sigma_{\rho}} \rightarrow \omega_{n-\ell} d L_{\Gamma}, \quad \text { and } \quad \rho^{\ell-n-1} d V_{\Sigma_{\rho}} \rightarrow \frac{\omega_{n-\ell}}{n+1-\ell} d L_{\Gamma}
$$

in the sense of measures. In particular,

$$
\rho^{\ell-n} d \mu_{\Sigma_{\rho}} \rightarrow \frac{\omega_{n-\ell}}{n+1-\ell} d L_{\Gamma}
$$

Proof. This follows from Fubini's theorem and the fact that the functions $w$ and $\Phi$ appearing in the parametrization of $\Sigma$ are uniformly controlled in $\mathcal{C}^{2, \alpha}$ as $\rho \rightarrow 0$.

On the other hand, we also have

Lemma 6.3. Let $E_{1}, \ldots, E_{n+1-\ell}$ be a local orthonormal frame for $N \Gamma$. Then, as $\rho \searrow 0$,

$$
\rho^{\ell-n}\left\langle\nabla_{N} X, N\right\rangle_{p} d A_{\Sigma_{\rho}} \rightarrow \frac{\omega_{n-\ell}}{n+1-\ell} \sum_{i}\left\langle\nabla_{E_{i}} X, E_{i}\right\rangle_{p} d L_{\Gamma}
$$

in the sense of measures.

Proof. As before, using Fubini's theorem and the uniform control on $w$ and $\Phi$, it suffices to check that this formula holds for the round sphere of radius $\rho$ in $\mathbb{R}^{n+1}$ as $\rho \rightarrow 0$, where again the formula is a standard computation. 
Now, multiply (6.2) by $\rho^{\ell-n}$ and let $\rho \rightarrow 0$. From these two lemmas, we conclude that

$$
\int\left(\operatorname{div} X-\sum_{i}\left\langle\nabla_{E_{i}} X, E_{i}\right\rangle\right) d L_{\Gamma}=0
$$

On the other hand, if $F_{1}, \ldots, F_{\ell}$ is a local orthonormal frame for $T \Gamma$, then

$$
\operatorname{div} X=\sum_{j=1}^{\ell}\left\langle\nabla_{F_{j}} X, F_{j}\right\rangle+\sum_{i=1}^{n+1-\ell}\left\langle\nabla_{E_{i}} X, E_{i}\right\rangle,
$$

so this last equation is equivalent to

$$
\int \sum_{j}\left\langle X, \nabla_{F_{j}} F_{j}\right\rangle d L_{\Gamma}=0 .
$$

Since the vector field $X$ is arbitrary, we conclude that the normal component of $\sum_{j} \nabla_{F_{j}} F_{j}$ is equal to 0 . This implies that the mean curvature of $\Gamma$ vanishes, i.e. that $\Gamma$ is minimal.

As already discussed at the beginning of this section, it would be much nicer to prove this theorem under less stringent hypotheses. We conclude by discussing this in more detail.

Suppose that there exist sequences of intervals $I_{k}=\left(\rho_{k}^{\prime}, \rho_{k}^{\prime \prime}\right)$ in $\mathbb{R}^{+}$with $\rho_{k}^{\prime}, \rho_{k}^{\prime \prime} \rightarrow 0$, such that for each $k$ and $\rho \in I_{k}$, there exists a CMC hypersurface $\Sigma_{\rho}$ isotopic to $\mathcal{T}_{\rho}(\Gamma)$ in $M \backslash \Gamma$. Suppose, furthermore, that this hypersurface satisfies:

a') the hypersurfaces $\left\{\Sigma_{\rho}\right\}_{\rho \in I_{k}}$ form a local foliation;

b') the mean curvature of $\Sigma_{\rho}$ equals $\frac{n-\ell}{n} \frac{1}{\rho}$;

c') there exists a constant $c>0$, independent of $k$ and $\rho \in I_{k}$, such that

$$
\Sigma_{\rho} \subset B_{\rho}(\Gamma):=\left\{q \in M^{n+1}: \quad \operatorname{dist}_{g}(q, \Gamma) \leq c \rho\right\} ;
$$

d') there exists a constant $c>0$, again independent of $k$ and $\rho \in I_{k}$, such that $\left|A_{\Sigma_{\rho}}\right| \leq c / \rho$.

We conjecture that these hypotheses alone are sufficient to conclude that $\Gamma$ is minimal. Indeed, it is possible to prove many of the necessary facts, but a few crucial ones seem much more difficult to obtain.

If we rescale $\Sigma_{\rho}$ from a point $p \in \Gamma$ by the factor $1 / \rho$, we obtain a family of CMC surfaces which are cylindrically bounded. We can obtain area 
bounds for these rescaled hypersurfaces, just as in [12], and so conclude that at least along subsequences, this family converges to a complete embedded cylindrically bounded hypersurface in $\mathbb{R}^{n+1}$. It is known [5] (and also [8]) that all such hypersurfaces must lie in the family of Delaunay unduloids $D_{\varepsilon}$; however, using that the $\Sigma_{\rho}$ are leaves of a local CMC foliation, we obtain a global bounded positive Jacobi field on the limiting surface, and it may be checked directly that in the Delaunay family, only the cylinder admits such a Jacobi field. Thus far, we have shown that, having fixed $p \in \Gamma$ and rescaling about this point, then some subsequence of these surfaces, say $\Sigma_{j}$, converges to a cylinder with axis parallel to the rescaled limit of $\Gamma$. The first difficulty is a standard one in the subject: if we knew the uniqueness of this limit, then we could straightaway conclude the existence of functions $w_{j}$ and $\Phi_{j}$ such that $\Sigma_{j}=\mathcal{T}_{\rho_{j}}\left(w_{j}, \Phi_{j}\right)$. We would also be able to conclude that

$$
\left\|w_{j}\right\|_{\mathcal{C}_{\rho}^{2, \alpha}}+\left\|\rho_{j}^{-1} \Phi_{j}\right\|_{\mathcal{C}_{\rho}^{2, \alpha}}=o(1) .
$$

However, we only obtain these bounds in $\mathcal{C}_{\rho}^{2, \alpha}$, not $\mathcal{C}^{2, \alpha}$. Because of this, we are unable to obtain bounds on the volume of $\Sigma$ of the form

$$
\int_{\Sigma_{\rho}} d A_{\Sigma} \leq C \rho^{n-\ell}
$$

on the other hand, it is quite straightforward to prove that $\int_{D\left(\Sigma_{\rho}\right)} d V_{\Sigma} \leq$ $C \rho^{n+1-\ell}$. In any event, we are only able to show that the second conclusion of Lemma 6.2 holds, and so, we are unable to take the weak limit of $d \mu_{\Sigma_{\rho}}$. The final difficulty arises in proving the analogue of Lemma 6.3, and this is the case because of the rather weak control we have for the derivatives of $w$ and $\Phi$ in the $\Gamma$ direction.

Plausibly, the most general theorem of this sort would involve a sequence of CMC hypersurfaces which are known to converge in Hausdorff distance, and perhaps satisfying hypotheses a') - d'). The conclusion should be that $\Sigma_{\rho}$ converge to a minimal submanifold $\Gamma$, of some dimension $\ell$, away from a set of Hausdorff measure smaller than $\ell$. For example, if $\ell=1$, it is quite conceivable that such a set of surfaces might converge to a broken geodesic (satisfying certain constraints at the break points).

\section{References.}

[1] F. Bethuel, G. Orlandi and Smets, Convergence of the parabolic Ginzburg-Landau equation to motion by mean curvature, C.R. Math. Acad. Sci. Paris 336 (2003) no. 9, 719-723. 
[2] F. Bethuel, G. Orlandi and Smets, Vortex rings for the Gross-Pitaevskii equation, J. Eur. Math. Soc. 6 (2004) no. 1, 17-94.

[3] A. Gray, Tubes, Addison-Wesley, Advanced Book Program, Redwood City, CA, 1990.

[4] G. Huisken and S.T. Yau, Definition of center of mass for isolated physical systems and unique foliations by stable spheres with constant mean curvature, Invent. Math. 124 (1996), no. 1-3, 281-311.

[5] N. Korevaar, R. Kusner and B. Solomon, The structure of complete embedded surfaces with constant mean curvature, J. Diff. Geom. 30, (1989), 465-503.

[6] J.M. Lee and T.H. Parker, The Yamabe problem, Bull. Amer. Math. Soc. (N.S.) 17 (1987), no. 1, 37-91.

[7] A. Malchiodi and M. Montenegro, Boundary concentration phenomena for a singularly perturbed elliptic problem, Comm. Pure Appl. Math. 55 (2002) no. 12, 1507-1568.

[8] R. Mazzeo, Recent advances in the global theory of constant mean curvature surfaces, in Contemporary Mathematics 350 Noncompact problems at the intersection of geometry, analysis and topology, Amer. Math. Soc. (2004).

[9] F. Mahmoudi, R. Mazzeo and F. Pacard, Constant mean curvature hypersurfaces condensing along a submanifold, Preprint, 2004.

[10] F. Pacard and M. Ritoré, From constant mean curvature hypersurfaces to the gradient theory of phase transitions, J. Diff. Geom. 64 (2003) no. $3,359-423$.

[11] R. Schoen and S.T. Yau, Lectures on Differential Geometry, Internat. Press, Cambridge, MA (1994).

[12] R. Ye, Foliation by constant mean curvature spheres, Pacific J. Math. 147 (1991), no. 2, 381-396.

[13] R. Ye, Constant mean curvature foliation: singularity structure and curvature estimate, Pacific J. Math. 174 (1996), no. 2, 569-587.

[14] R. Ye, Foliation by constant mean curvature spheres on asymptotically flat manifolds, in Geometric analysis and the calculus of variations 369383, Internat. Press, Cambridge, MA (1996). 
[15] B. White, The space of minimal submanifolds for varying Riemannian metrics, Indiana Univ. Math. J. 40 (1991), no. 1, 161-200.

[16] T.J. Willmore, Riemannian Geometry, Oxford Univ. Press. NY. (1993).

Department of Mathematics

STANFORD UNIVERSITY

STANFORD, CA 94305

USA

E-mail address: mazzeo@math.stanford.edu

Université Paris XII Val de Marne and Institut Universitaire de FRANCE

Laboratoire D'Analyse et de Mathématiques Appliquées

61 Avenue du GÉnéral de Gaulle, 94010

Créteil Cedex

PARIS-FRANCE

E-mail address: pacard@univ-paris12.fr

Received August 8, 2003. 